\title{
Concentración/Especialización económica en el Ecuador
}

\section{Concentration/Specialisation in Ecuador}

\author{
Carlos Almeida* \\ Lilia Quituisaca-Samaniego** \\ Luis Antamba*** \\ *Departamento de Matemática, Escuela Politécnica Nacional, Quito - Ecuador \\ **Sociedad Ecuatoriana de Estadística, Quito-Ecuador \\ ***Riesgos de crédito, ICESA S.A, Quito - Ecuador
}

HTTPS://DOI.ORG/10.22370/PE.2019.8.2528

\section{Resumen}

En este trabajo se explora la heterogeneidad de la estructura económica de la pequeña empresa en el Ecuador, para relacionar las actividades económicas en una unidad geográfica, así, como la distribución de los pesos que representa cada sector económico (manufactura, comercio y servicios). Se utiliza la distancia de Hellinger entre distribuciones para comparar las estructuras económicas y las distribuciones geográficas de los diferentes sectores y actividades de la economía, según la clasificación CIIU 4.0. Para caracterizar una economía, se utiliza el número de trabajadores que pertenecen a un sector económico en una unidad geográfica. Finalmente, se utiliza estas distancias, para hacer agrupaciones geográficas, que conserven continuidad territorial. La fuente de datos para el análisis está constituido por los registros del Censo Nacional Económico (CENEC, 2010) del Instituto Nacional de Estadística y Censos (INEC).

Palabras clave: Concentración económica, especialización económica, agrupación territorial Códigos JEL: C60, L60, L70, L80

\section{Abstract}

This paper explores the heterogeneity of the economic structure of the small dam in Ecuador in order to relate economic activities in a geographical unit, as well as the distribution of the weights represented by each economic sector (manufacture, trade and services). The Hellinger distance between distributions is used to compare the economic structures and geographic distributions of the different sectors and activities of the economy, according to the ISIC 4.0 classification. To characterise an economy, the number of workers belonging to an economic sector in a geographical unit is used. Finally, these distances are used to make geographical groupings that preserve territorial continuity. The data source for the analysis is constituted by the records of the National Economic Census (CENEC, 2010) of the National Statistics and Census Institute (INEC).

Keywords: Economic concentration, economic specialization, territorial grouping

*carlos.almeidar@epn.edu.ec

*1ilia.quituisaca.samaniego@gmail.com

*luis_antamba@hotmail.com 


\section{INTRODUCCIÓN}

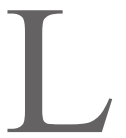

a división política y administrativa del Ecuador, es muy heterogénea en muchos sentidos, por ejemplo, hay cantones evidentemente empresas) y otros de gran concentración humana, especialmente las ciudades principales y capitales de provincia.

Las grandes aglomeraciones urbanas generan geográficamente dinámicas territoriales que deben ser caracterizados de forma adecuada. Las interrelaciones de los diferentes sectores de la economía generan a su vez concentración geográfica de determinados grupos e influencian en la demografía, esta demografía genera de la misma forma necesidades que dinamizan otros sectores.

Estudios enfocados a caracterizar la estructura industrial por regiones se han llevado a cabo en Chile y Argentina $(2,3,6$;) en estos países, la importancia de los sectores industriales es mucho mayor que en Ecuador, donde la importancia relativa de la agricultura y la ganadería es mucho mayor (10). Sin embargo, en el país se ha considerado un estancamiento del sector primario de la economía ( $\sin$ el petróleo), es decir la agricultura, ganadería, silvicultura, que en 1995 representaba el $13 \%$ del PIB y al 2004 cae al $6 \%$. Dicha tendencia contractiva se estabiliza hasta el 2012 (participación del $6 \%$ ) (9). El presente estudio aplica conceptos para toda la economía nacional y no sólo enfocada al sector industrial.

El término concentración económica lo reservamos a la idea de que las actividades económicas en general se distribuyen geográficamente en ciertas unidades geográficas, en el caso de Ecuador, se piensa que Quito, Guayaquil o Cuenca concentran buena parte de la economía (12).

En revancha la especialización económica toma cuenta del hecho que determinadas regiones, por cuestiones de ventajas comparativas o de tradición se 'especializan' en una u otra actividad (11).

Tomando en cuenta el tamaño de las economías locales, se compara estas a través de una distancia, y se intenta agrupar estas unidades geográficas conservando continuidad territorial, es decir sólo se agrupan entre vecinos. Para lograr una agrupación en este sentido, se 
calcula una disimilitud que es igual a la distancia para las regiones Tomando en cuenta el tamaño de las economías locales, se compara estas a través de una distancia, y se intenta agrupar estas unidades geográficas conservando continuidad territorial, es decir sólo se agrupan entre vecinos. Para lograr una agrupación en este sentido, se calcula una disimilitud que es igual a la distancia para las regiones vecinas, y penalizada por un valor relativamente grande si las regiones no son vecinas, luego se calcula el árbol de peso mínimo con estas disimilitudes y se separa las unidades geográficas (cantones) suprimiendo el eje de mayor peso de este árbol de peso mínimo, se aplica ese mismo algoritmo de manera recursiva para obtener un dendrograma que nos ilustra la forma como las regiones se pueden ir agrupando (separando) paulatinamente. Para detalles sobre el algoritmo de peso mínimo ver (8).

También se comparan los sectores económicos usando las misma distancia, pero esta vez entre las distribuciones geográficas de los sectores económicos. Se busca agrupar sectores económicos que tienen distribuciones geográficas similares (o 'cercanas'), permitiendo en cierta medida suponer que existe una simbiosis importante entre estos sectores.

Este trabajo está estructurado de la siguiente manera: en la Sección 2 se presenta el marco teórico que introduce los conceptos y describe la metodología de cálculo de los diferentes elementos que se usa, en la Sección 3 se hace la aplicación de esta metodología y se discuten los resultados, se finaliza con la Sección 4 de conclusiones que se derivan del presente estudio. Para complementar, se añaden anexos que sirven para soportar la discusión de resultados.

\section{MarCo teÓRICO}

\subsection{TABLAS DE CONTINGENCIA}

A partir de datos que nos representen la talla de las empresas (número de empleados, volumen de ventas, etc.) ubicados geográficamente y por sector, encontrar métricas que nos permitan comparar a su vez los sectores económicos de un lado y las regiones (o unidades geográficas) por otro lado. 
El tamaño o importancia de un sector económico, en el presente estudio está dado por el número de empleados que tiene ese sector, se puede imaginar otras formas de mediresto, comola cifra denegocios o las ganancias declaradas, o el capital social, etc., pero el número de empleados parece ser un parámetro razonable (7).

En (6) utilizan métricas o distancias (divergencias) entre distribuciones condicionales. Más concretamente, en una tabla de contingencia de número de empleados por sectores económicos y regiones geográficas, se pueden estimar las distribuciones condicionales, y se puede definir deferentes métricas (o divergencias), por ejemplo: distancia de Hellinger o divergencia de Kullback-Leibler (o su versión simétrica), o la distancia Chi-cuadrado. En (1) se tiene una revisión más detallada en tablas de contingencia.

Para el presente estudio, y por fijar notaciones, se usa lo siguiente: Sea $N=\left(n_{\mathrm{ij}}: 1 \leq i \leq I ; 1 \leq j \leq J\right)$ una tabla de contingencia, donde $n_{\mathrm{ij}}$ es el número de empleados ocupados en la unidad geográfica (cantón) $i$ en el sector (rama) económico $j$.

Además, se definen las sumas marginales:

$$
N_{i \bullet}:=\sum_{j=1}^{J} n_{i j}, \quad N_{\bullet j}:=\sum_{i=1}^{I} n_{i j} \text { y } \quad N_{\bullet .}:=\sum_{i=1}^{I} \sum_{j=1}^{J} n_{i j} .
$$

Por convención de aquí en adelante se utilizará $i$ para las unidades geográficas (cantones o agrupaciones de cantones), y $j$ para los sectores o ramas económicas o agrupaciones de estas según corresponda.

En general, una tabla de contingencia es la realización de una distribución multinomial

$$
M N\left(N . .,\left(P_{i j}: 1 \leq i \leq I, 1 \leq j \leq J\right)\right),
$$

donde $p_{i j}$ es la probabilidad de que un empleado tomado al azar en la población de referencia trabaje en la región $i$ en una empresa del sector $j$. Entonces se tendrá que $\Sigma_{i, j} p i j=1$, y $p_{i j} \geq 0$; se definen 
los probabilidades marginales por:

$$
P_{i} \bullet:=\sum_{j=1}^{J} P_{i j}, \quad P_{\bullet j}:=\sum_{i=1}^{I} P_{i j},
$$

que se interpretan como la probabilidad que un empleado elegido al azar sea del cantón $i$ o del sector $j$ respectivamente. Las probabilidades condicionales están dadas por:

$$
\left.P_{i}\right|_{j}:=\frac{P_{i j}}{P_{\bullet j}},\left.\quad P_{j}\right|_{i}:=\frac{P_{i j}}{P_{i}}
$$

Estas probabilidades pueden interpretarse, en el caso de $\left.P_{i}\right|_{j}$ como la proporción de empleados del sector $j$ que están en la región $i$. y recíprocamente, $\left.P_{j}\right|_{i}$ es la proporción de empleados en la región $i$ que trabajan en el sector $j$.

Consistentemente, por el método de máxima verosimilitud, a partir de los datos, estas probabilidades se estiman por:

$$
\begin{gathered}
\hat{P}_{i j}=\sum_{j=1}^{J} \frac{N_{i j}}{N_{\bullet \bullet}} \\
\hat{P}_{\bullet \bullet}=\sum_{j=1}^{J} \hat{\hat{p}_{i j},} \quad \hat{P}_{\bullet j}=\sum_{i=1}^{I} \hat{P}_{i j} \\
\left.\left.\hat{P}_{i}\right|_{j=\frac{N_{i j}}{N_{\bullet j}}} \quad \hat{P}_{j}\right|_{i}:=\frac{N_{i j}}{N_{i} \bullet}
\end{gathered}
$$

\subsection{MÉTRICA DE HELLINGER}

Luego, los medidas de concentración/especialización estarán relacionados con la "distancia" de estas distribuciones condicionales a las marginales, pues esta da cuenta del comportamiento global de todo el país, además, dependiendo de que si tratamos con filas o columnas compararemos sectores económicos o unidades geográficas.

La métrica usada en este estudio es la distancia de Hellinger, 
esta distancia a-priori tiene dos ventajas, la primera es que al usar la raíz cuadrada, mejora el impacto de las pequeñas diferencias y aumenta el impacto de las grandes; la segunda ventaja es que esta distancia está acotada por $\sqrt{2}$. En general, entre dos distribuciones de probabilidad $\mathrm{P}$ y $\mathrm{Q}$ con densidades con respecto a una medida dominante $\mu$ dadas por $f$ y $g$ respectivamente, se define la distancia de Hellinger por:

$$
d H(P, Q)=\int(\sqrt{f}-\sqrt{g})^{2} d \mu .
$$

Observe que este valor siempre está en el intervalo $[0,2]$ para cualquier par de distribuciones de probabilidad.

En el caso de distribuciones discretas, estas son dominadas por la medida de conteo y entonces esta distancia viene dada por:

$$
d H(P, Q)=\sum_{i \in I}\left(\sqrt{p}_{i}-\sqrt{q}_{i}\right)^{2}
$$

$\operatorname{con} \sum_{p_{i}}=\sum_{q_{i}=1}$ y todos no negativos.

Luego, definimos la distancia entre dos sectores económicos como la distancia de Hellinger entre las distribuciones condicionales respectivas, estará dada por:

$$
d s(\text { Sector } U, \text { Sector } V)=\sum_{i \in \text { Canton }}\left(\sqrt{\left.p_{i}\right|_{j}=U}-\sqrt{\left.p_{i}\right|_{j}=V}\right)^{2},
$$

Y la correspondiente distancia entre dos unidades geográficas (cantones) estará dada por:

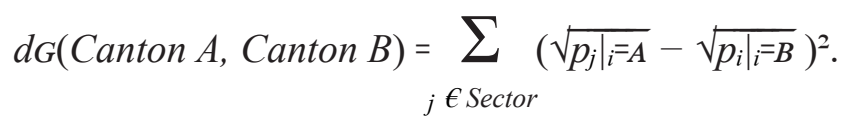

\subsection{CONSTRUCCIÓN DE INDICES}

La construcción de índices de concentración/especialización, se hace por comparación a la distribución nacional; más específicamente, 
el índice de especialización de un Cantón A está dada por:

$$
d G(\text { Canton A, Pais })=\sum_{j \in \text { Sector }}\left(\sqrt{\left.p_{j}\right|_{i}=A}-\sqrt{p_{\bullet j}}\right)^{2} .
$$

Así mientras más alto sea el valor de esta distancia, más especializada será la economía de ese cantón.

También se puede comparar las diferentes ramas o sectores económicos a la estructura global del país:

$$
d s(\text { Sector V, Pais })=\sum_{i \in \text { Canton }}\left(\sqrt{p_{i} \mid j=V}-\sqrt{p_{i \bullet}}\right)^{2} .
$$

Es claro que un valor de 0 para estos valores se debe a que el sector económico (resp. la unidad geográfica) tiene una estructura igual a la media nacional, por lo que el sector (resp. cantón) no estará concentrado (resp. especializado), contrariamente valores altos significan concentración (resp. especialización).

A partir de las distancias descritas se construye dos índices de especialización, que se definen como medias ponderadas de las mismas.

Para el Índice 1 (Sectores/cantones), se tome la media de las distancias ponderada por el tamaño de economía de cada unidad geográfica (Cantón):

$$
I_{n} d_{1}=\sum_{i \in \text { Canton }} \mathrm{dG}_{(i, \text { Pais })} p_{i}
$$

Este índice también puede calcularse para provincias u otras agrupaciones territoriales exactamente de la misma manera.

Para el Índice 2 (Cantones/Sectores), la media ponderada se realiza por sectores económicos:

$$
\operatorname{Ind}_{2}=\sum_{j} d_{\text {Sector }} d s(j, \text { Pais }) p \bullet j
$$




\subsection{Agrupaciones}

De sectores o ramas económicas A partir de la matriz de distancias entre las distribuciones condicionales $\mathrm{P}_{i} \mid j$, que se interpreta como la distribución geográfica por cada sector económico, se hace una clasificación (eventualmente utilizando el algoritmo del árbol de peso mínimo) y se obtiene agrupaciones algo homogéneas de sectores económicos. En este punto se definen tres estrategias para comparar y posteriormente hacer las agrupaciones geográficas:

Alta agrupación a partir de los datos, se consideran los tres macro sectores tradicionales: Manufactura, Comercio y Servicios.

Ninguna agrupación Se considera los datos en todos los sectores económicos de los que se tiene datos (206 sectores, ver descripción en el Apéndice C).

Agrupación media Se agrupa de forma automática los sectores, se consideran (20 grupos de sectores con la descripción en el Apéndice). Geográfica En cada unos de las agrupaciones, las mismas que están dadas por sectores, se redefine la tabla de contingencia y se recalculan las distancias entre cantones.

Con las distancias y considerando una penalidad suficientemente grande si los cantonesno son vecinos, se trata estas como disimilitudes y mediante el algoritmo del árbol de peso mínimo, se logra una separación recursiva que nos permite separar en regiones que conservan continuidad territorial.

\section{ApliCACIÓN}

Se desarrolla la aplicación de lo descrito en el marco teórico a los datos de Censo Nacional Económico del 2010, primeramente, hay consideraciones hechas para filtrar los datos a condiciones de aplicación de la metodología descrita, especialmente en lo referente a sectores económicos o cantones que no registran datos o como en el caso de Galápagos, por su situación geográfica y status particular no se lo considera para este estudio. 


\subsection{DESCRIPCIÓN DE LOS DATOS}

La fuente de datos está constituido por los registros del Censo Nacional Económico (CENEC) del 2010. Se consideran las variables detalladas en la Tabla 1, algunas propias de la encuesta y otras creadas conforme la necesidad de caracterización, especialmente la recodificación de modalidades, por ejemplo: la clasificación de empresas $^{1}$. De la base de datos CENEC 2010 se eliminan registros que cumplen las siguientes condiciones:

- Las provincias que representan hasta el 0,5\% a nivel nacional, en nuestro caso Galápagos y Zonas no delimitadas

- Los establecimientos que no recogen información de personal ocupado.

- Las medianas y grandes empresas

- Los establecimientos que pertenecen a otros (Figura 5); porque representan menos del 0,5\%.

Luego de aplicar las condiciones anteriores, quedan $\mathbf{4 7 4 . 4 1 5}$ registros de establecimientos económicos. De esta manera, las variables de caracterización a utilizarse son:

\begin{tabular}{lll}
\hline \hline Descripción & Categorías iniciales & Categorías de estudio \\
\hline \hline Ubicación geográfica & 223 cantones & 218 cantones \\
\hline \multirow{3}{*}{ Sectores económicos } & Manufactura & Manufactura \\
& $\begin{array}{l}\text { Comercio } \\
\text { Servicios }\end{array}$ & Servicios \\
& Otros & \\
\hline & no informa & \\
& $1-9$ & $1-9$ \\
& $10-49$ & $10-49$ \\
& $50-99$ & $50-99$ \\
Personal ocupado & $100-199$ & $100-199$ \\
& $200-499$ & $>=200$ \\
\hline Actividad principal económica $\left(\mathrm{CIIU}^{4}\right)$ & 500 y mas & \\
\hline
\end{tabular}

Tabla 1: Variables de estudio. Fuente: INEC - CENEC 2010. Elaboración propia

Brevemente podemos observar que a nivel nacional, según el número de establecimientos tenemos:

- Por subsector tenemos que el 55,14\% corresponde a comercio, el 35,14\% corresponde a servicios y el 9,72\% corresponde a manufactura. 
- Por ubicación geográfica, alrededor del $50 \%$ de establecimientos económicos está en las provincias de Pichincha, Guayas, Azuay, Tungurahua.

- Por personal ocupado el 96,90\% está en el primer estrato (1-9 trabajadores), de los cuales el 66,65\% está compuesto hasta de dos trabajadores.

\section{2. ÍNDICES DE CONCENTRACIÓN/ESPECIALIZACIÓN}

La aplicación de las formulas (1) y (2), nos permiten saber en que medida los cantones son diferentes de la media nacional en estructura económica ( $\mathrm{dG}_{\mathrm{G}}$ y en que medida es diferente la distribución geográfica de una rama o actividad económica con la distribución global de la economía del país (ds).

\section{3. ÍNDICE DE CONCENTRACIÓN}

Como referencia se tienen medidas globales que dan una idea de heterogeneidad global del país en lo referente a la concentración/ especialización económica en el país, también se calcula por regiones.

A continuación, la Tabla 2 muestra un resumen de los cantones con el mayor y menor índice de concentración laboral con respecto a la media nacional. El índice de concentración de todos los cantones puede verse en el Apéndice C- Tabla11

\begin{tabular}{|c|c|c|c|}
\hline Orden & Cantón & Índice concentración $\left(d_{s}\right)$ & Peso \\
\hline 1 & Guayaquil & 0.0949 & 0.1746 \\
2 & Quito & 0.1056 & 0.2020 \\
3 & Riobamba & 0.1192 & 0.0243 \\
4 & Manta & 0.1238 & 0.0181 \\
$\vdots$ & $\vdots$ & $\vdots$ & $\vdots$ \\
7 & Cuenca & 0.1459 & 0.0569 \\
$\vdots$ & $\vdots$ & $\vdots$ & \\
215 & Penipe & 0.5195 & 0.0002 \\
216 & Olmedo & 0.5635 & 0.0001 \\
217 & Sucumbíos & 0.5645 & 0.0001 \\
218 & Aguarico & 0.7616 & 0.0000 \\
\hline
\end{tabular}

Tabla 2: Índice de concentración económica con relación a la media nacional (0.1635) 
Los cantones Guayaquil y Quito tienen una mayor concentración laboral con relación a la media nacional; de la misma manera, Sucumbíos y Aguarico son los cantones menos parecidos a la media nacional.

La cercanía de Quito y Guayaquil a la estructura nacional se explica por el peso que estas ciudades tienen en la economía, pues representan conjuntamente cerca del $30 \%$ de la economía nacional. Los casos de Riobamba y Manta resultarían interesantes de analizar más a profundidad.

El caso de los cantones que son muy diferentes (Penipe, Olmedo, Sucumbíos y Aguarico), sus estructuras económicas son bastante diferentes a la media nacional, pero su peso en la economía nacional es muy marginal.

\section{4. ÍNDICE DE ESPECIALIZACIÓN}

El cálculo del índice de especialización se resume en la Tabla 3, destacándose el hecho que actividades de servicio y de comercio, que tienen un peso relativamente importante, estén más cerca de la media nacional, y actividades que son realmente marginales se distribuyen geográficamente muy diferentes la distribución geográfica de la economía ecuatoriana. El índice de especialización de todas las actividades económicas (CIIU Rev. 4.0 a 3 dígitos) puede verse en el Apéndice C- Tabla12

\begin{tabular}{|r|l|c|c|}
\hline Orden & \multicolumn{1}{|c|}{ Actividad económica } & Índice (especialización) & Peso \\
\hline 1 & I561 - Actividades de Restaurantes ... & 0.0558 & 0.0912 \\
2 & S960 - Servicios Personales (Otras) & 0.0643 & 0.0364 \\
3 & G475 - Venta al Por menor (Otros enseres domésticos) & 0.0645 & 0.0380 \\
4 & G477 - Venta al Por menor (Otros prod. comercio especializado) & 0.0656 & 0.0935 \\
$\vdots$ & $\vdots$ & $\vdots$ & $\vdots$ \\
203 & C303 - Fabricación de Aeronaves & 0.8133 & 0.0000 \\
204 & E382 - Tratamiento y Eliminación de desechos & 0.8213 & 0.0000 \\
205 & N813 - Actividades de Investigación & 0.8309 & 0.0000 \\
206 & D352 - Fabricación de Gas ... & 0.8649 & 0.0000 \\
\hline
\end{tabular}

Tabla 3: Índice de especialización con relación a la media nacional (0.1452) 


\subsection{REDUCCIÓN (AGRUPACIÓN) DE RAMAS ECONÓMICAS}

En un extremo del análisis disponemos de tres grandes sectores, manufactura, comercio y servicios, y en el otro extremo una desagregación en 206 ramas o actividades económicas, se hace una agrupación territorial, considerando estos dos casos, como de gran agrupación y sin agrupación de ramas económicas respectivamente y se considera un tercer caso que sería de agrupación media.

Para este último caso se considera la distancia entre sectores $(d s)$ y se hace una clasificación (en cluster), la aplicación del algoritmo nos el dendrograma de la Figura 1.

Se fija el nivel a 20 grupos de sectores económicos, cuya composición se detalla en el Apéndice D.

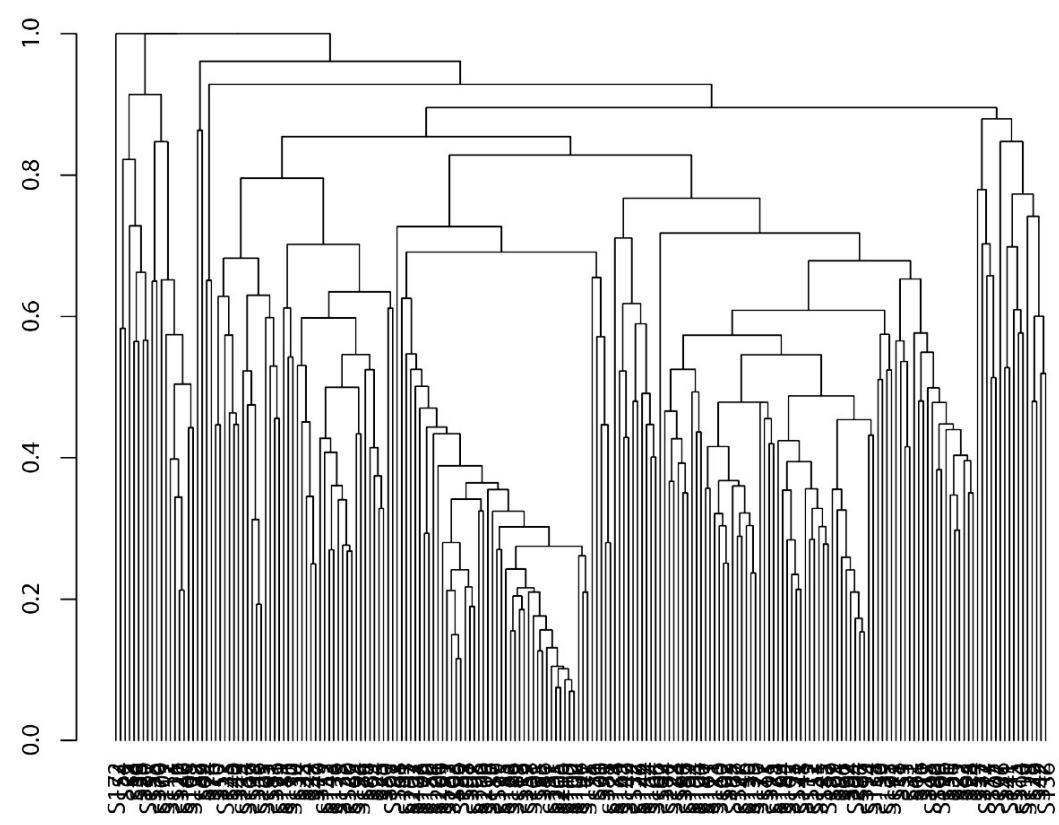

Figura 1: Dendrograma para la agrupación de ramas o sectores económicos. INEC - CENEC 2010. Elaboración propia 


\subsection{Agrupación GEOGRÁFICA}

Para cada caso de agrupación de ramas económicas se aplica la separación geográfica con continuidad territorial, para esta se considera la distancia entre regiones geográficas $\left(d_{G}\right)$ y se considera una penalidad suficientemente grande para los cantones que no son vecinos, recursivamente se calcula el árbol de peso mínimo (que convenientemente contendrá solo ejes entre cantones vecinos), y se separa los cantones por el eje de mayor peso en ese árbol de peso mínimo.

Como resumen de resultados se tiene:

\subsubsection{SIN AGRUPACIÓN DE SECTORES ECONÓMICOS}

Considerando los datos de las 206 ramas económicas y también aplicando el mismo esquema para comercio, manufactura y servicios por separados se tiene los siguientes resultados.

\begin{tabular}{|c|c|c|c|c|c|c|c|}
\hline \multicolumn{2}{|c|}{ Nacional } & \multicolumn{2}{|c|}{ Comercio } & \multicolumn{2}{|c|}{ Manufactura } & \multicolumn{2}{|c|}{ Servicios } \\
\hline No. Grupos & \begin{tabular}{|l} 
No. Cantones \\
\end{tabular} & No. Grupos & No. Cantones & No. Grupos & \begin{tabular}{|l|} 
No. Cantones \\
\end{tabular} & No. Grupos & \begin{tabular}{|l|} 
No. Cantones \\
\end{tabular} \\
\hline 1 & 95 & 1 & 43 & 1 & 56 & 1 & 91 \\
\hline 2 & 5 & 1 & 23 & 1 & 20 & 1 & 6 \\
\hline 1 & 3 & 1 & 11 & 1 & 19 & 1 & 3 \\
\hline 4 & 2 & 1 & 9 & 1 & 4 & 11 & 2 \\
\hline & & 1 & 6 & 4 & 3 & & \\
\hline & & 2 & 5 & 5 & 2 & & \\
\hline & & 2 & 3 & & & & \\
\hline & & 9 & 2 & & & & \\
\hline 8 & 116 & 17 & 126 & 4 & 121 & 14 & 122 \\
\hline \multicolumn{8}{|c|}{ No Agrupados (Cantones) } \\
\hline & 102 & & 92 & & 97 & & 96 \\
\hline
\end{tabular}

Tabla 4: Agrupación por cantones y ramas económicas. Fuente CENEC Elaboración propia.

En la tabla 4, se muestra la agrupación de cantones por ramas de actividad, en la cual se encuentran el número de grupos y el número de cantones correspondientes a cada grupo en orden de agrupación (por cantones); si nos referimos a Nacional, significa que: existen 93 cantones en un grupo, 2 grupos de 5 cantones cada uno, un grupo de 3 cantones y 2 grupos con 4 cantones; en total se tienen 8 grupos distintos que contienen a 116 cantones, dejando un total de 112 cantones sin ningún tipo de agrupación. Los cantones que pertenecen a cada uno de estos grupos se los puede ver en la Tabla 7. 
Nacional

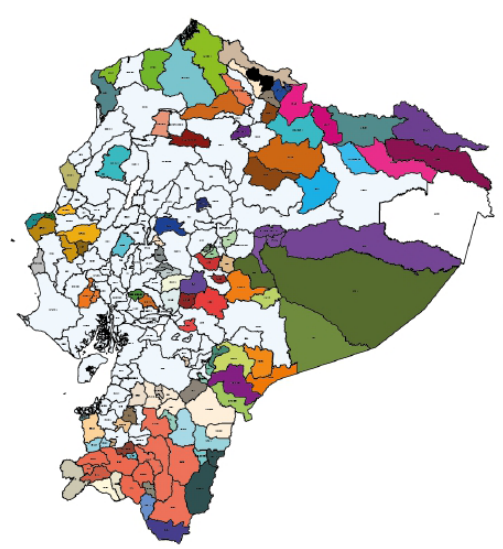

Comercio

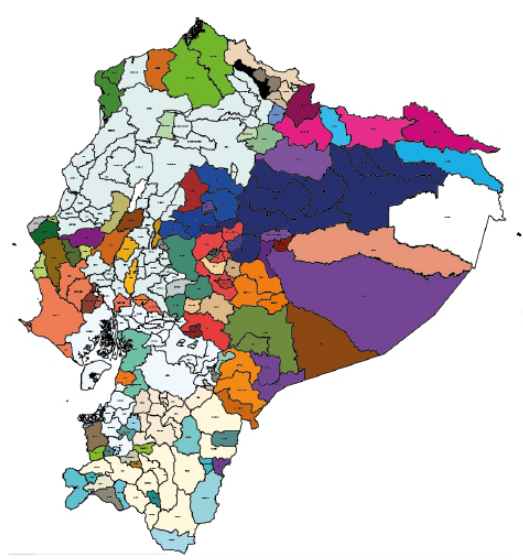

Manufactura

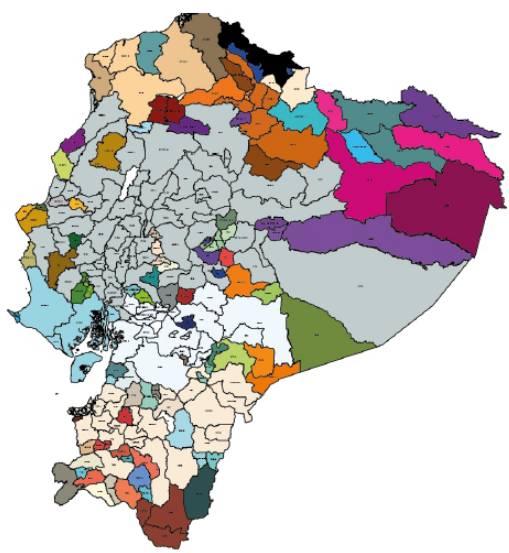

Servicios

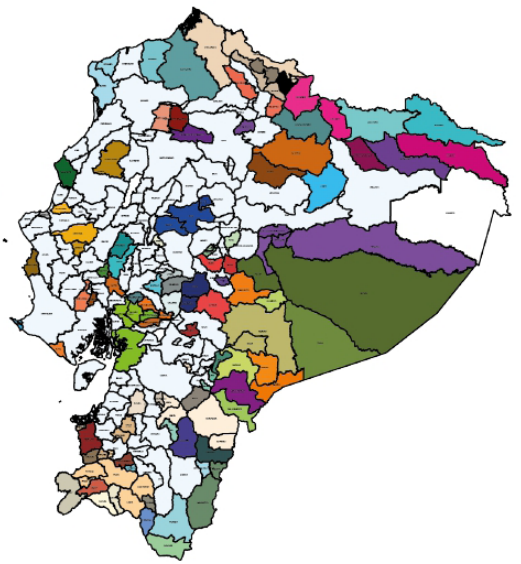

Figura 2: Separación territorial por considerando alta agrupación de ramas económicas y por regiones. Fuente: Elaboración propia Fuente: INEC - CENEC 2010. Elaboración propia. La separación territorial resultante, se detalla en el Apéndice B 


\subsubsection{Alta AGRUPACión De SeCTORES ECONÓMiCOS}

Se considera únicamente los tres macros-sectores agrupados: Manufactura, Comercio y Servicios, y se hace la separación territorial como se muestra en el siguiente mapa.

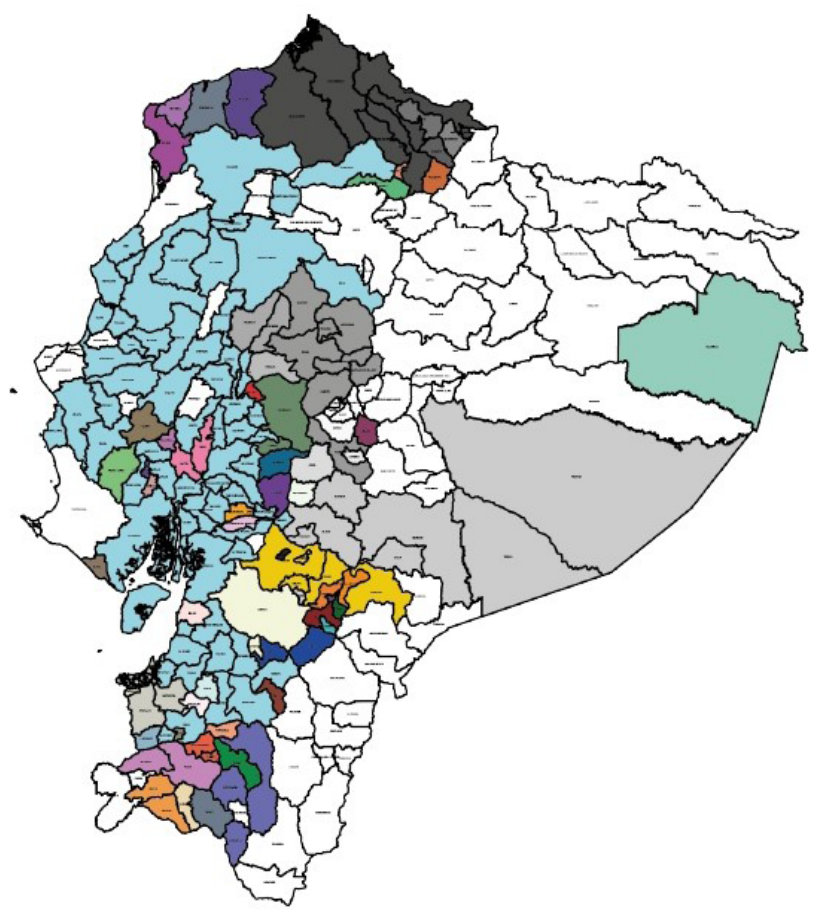

Figura 3: Agrupación por manufactura, comercio y servicios agrupados a nivel nacional. INEC- CENEC 2010. Elaboración propia.

Cuando existe menor desagregación, los resultados evidencian agrupaciones geográficas muy diferentes, mostrando que la metodología aplicada es sensible al nivel de desagregación de la información, en el futuro debería construirse alguna forma de evaluar la pérdida de información relacionada con la agrupación y así encontrar una descripción de la estructura económica más estable. 


\subsubsection{Media AGRUPACiÓN DE SECTORES ECONÓMICOS}

Considerando 20 grupos de actividades económicas que resultan de la clasificación de ramas resultante en la Sección 3.5, (ver Apéndice D por detalles de esta agrupación). Los resultados en este caso de la separación territorial son:

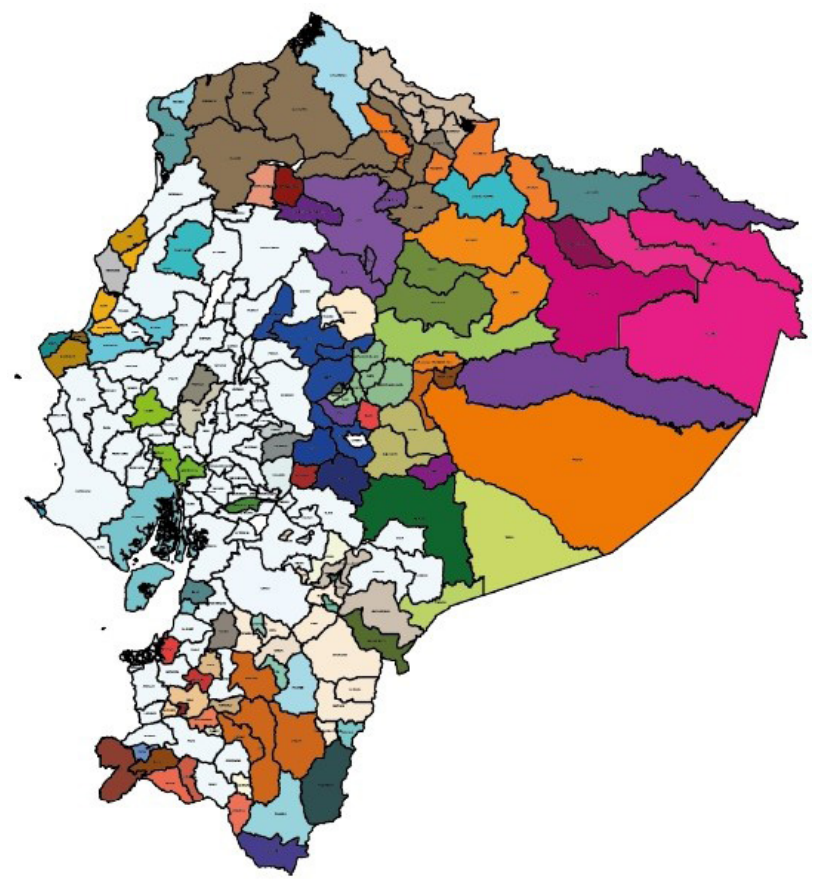

Figura 4: Agrupación Media de sectores económicos. INEC - CENEC 2010. Elaboración propia. 


\section{Conclusiones}

- La construcción de índices basados en las distribuciones condicionales permite comparar regiones geográficas y/o sectores económicos independientemente del tamaño o peso que estos puedan tener en la economía nacional.

- EL algoritmo implementado con el árbol de peso mínimo permite de forma fácil hacer agrupaciones territoriales conservando continuidad territorial, pues solo puede asociar vecinos.

- Ya en la aplicación a los datos del Censo Económico Nacional 2010, la gran influencia que tienen los centros urbanos en la economía del país, evidenciando que la separación entre los dos grandes centros económicos del país (Quito y Guayaquil) no se presenta rápidamente a ningún nivel de agregación estudiado.

- Hace falta estudios más profundos, podría extenderse a empresas más pequeñas, de forma que se pueda visualizar mejor las interacciones locales.

- En todo el estudio no se ha considerado el problema de la calidad de los datos, sin embargo, es recomendable su estudio futuro.

\section{REFERENCIAS}

(1) A. Agresti, Categorical Data Analysis, Wiley Series in Probability and Statistics, Wiley-Interscience, 2nd ed., 2002.

(2) M. BADIA-Miró, El crecimiento industrial y concentración de la actividad económica en chile, 1894-1967, pp. 1-41. Universitat Oberta de Catalunya.

(3) J. G. BRIda, S. London, AND M. Rojas, Una aplicación de los árboles de expansión mínima y árboles jerárquicos al estudio de la convergencia interregional en dinámica de regímenes, Revista de Métodos Cuantitativos para la Economía y la Empresa, 15 (2013), pp. 3 - 28.

(4) C. Comunidad Andina, Resolución 1260: Disposición Técnica para la Transmisión de Datos de Estadísticas de PYME de los Países Miembros de la Comunidad Andina, Secretaría General Comunidad Andina, (2009). 
(5) I. N. De Estadística y Censos, Clasificación Nacional de Actividades Económicas (CIIU REV. 4.0), Unidad de Análisis de Síntesis, (2012).

(6) C. HAEDO AND M. MouchaRT, Specialized agglomerations with areal data: model and detection, CORE Discussion Papers 2013060, Université catholique de Louvain, Center for Operations Research and Econometrics (CORE), 2013.

(7) INEGI, Micro, pequeña, mediana y gran empresa: Estratificación de los establecimientos, (2009), pp. 9-150. Censos Económicos 2009.

(8) Z. LOKTER AND D. PELEG, Minimum-weight spanning tree construction in o( $\log \log n)$ communication rounds, 35 (2005), pp. 120-131. SIAM J. Comput.

(9) J. Rodriguez, Un sector que debe crecer, Ekos Negocios, (2012), pp. $48-49$.

(10) O. Sotomayor, A. Rodríguez, And M. Rodrigues, Competitividad, sostenibilidad e inclusiø'n social en la agricultura: Nuevas direcciones en el diseño de políticas en América Latina y el Caribe, CEPAL, 2011.

(11) T. TANDAZo-Arias, La actividad económica del ecuador: Una visión desde la economía espacial, (2012), pp. 1-24. Departamento de Economía.

(12) — L L L regionalización económica del ecuador, (2012), pp. 151157. Instituto de Investigaciones Económicas. 


\section{Apéndices}

\section{A. Insumos}

\begin{tabular}{r|cccc}
\hline Variables & Estrato I & Estrato II & Estrato III & Estrato IV \\
\hline $\begin{array}{r}\text { Personal Ocupado } \\
\text { Valor Bruto }\end{array}$ & $1-9$ & $10-49$ & $50-99$ & $100-199$ \\
de las Ventas Anuales (US\$) & $<=\$ 100.000$ & $\$ 100.001$ a $\$ 1^{\prime} 000.000$ & $\$ 1.000 .001$ a $\$ 2.000 .000$ & $\$ 2.000 .001$ a $\$ 5.000 .000$ \\
\hline
\end{tabular}

Tabla 5: Variable de clasificación de empresas, según estratos. Fuente: Comunidad Andina [4]
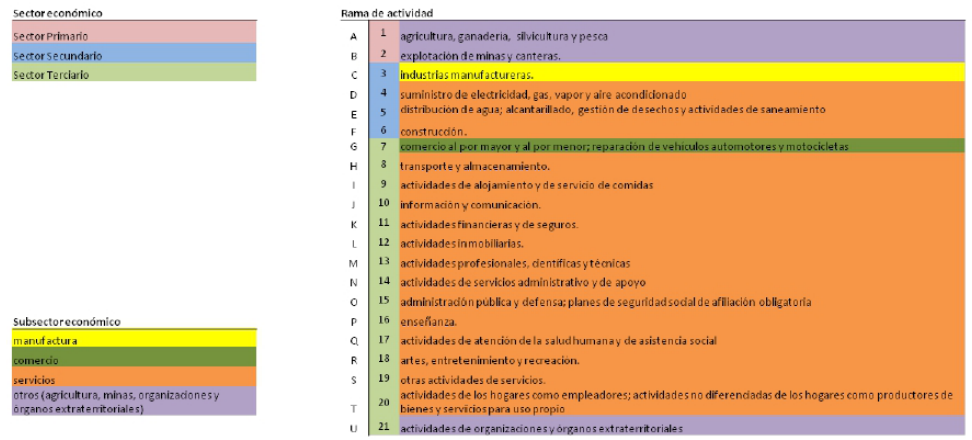

Figura 5: Clasificación Nacional de Actividades Económicas CIIU Rev. 4.0. Fuente: INEC Análisis de Síntesis 2012 [5]. Elaboración propia. 


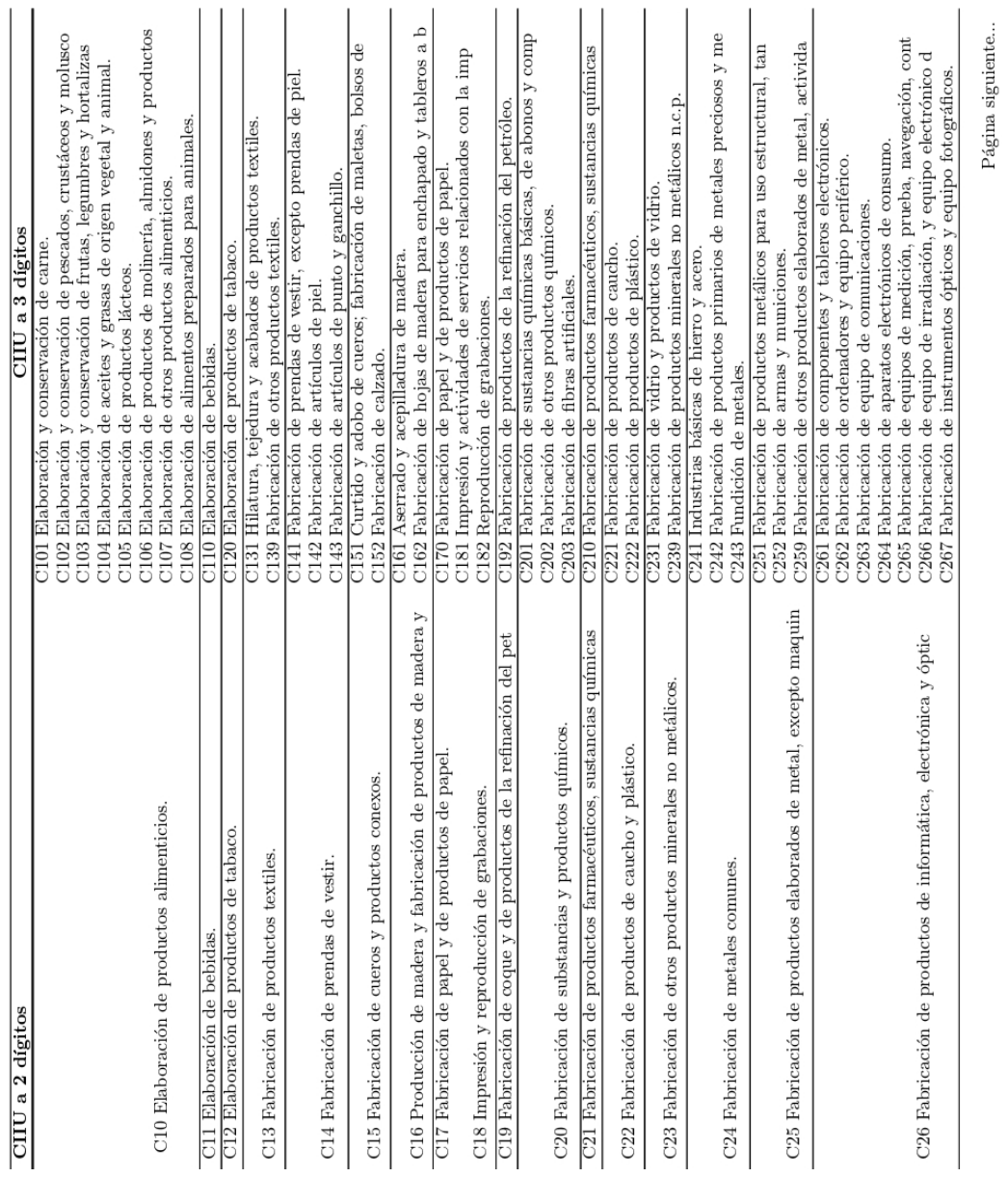




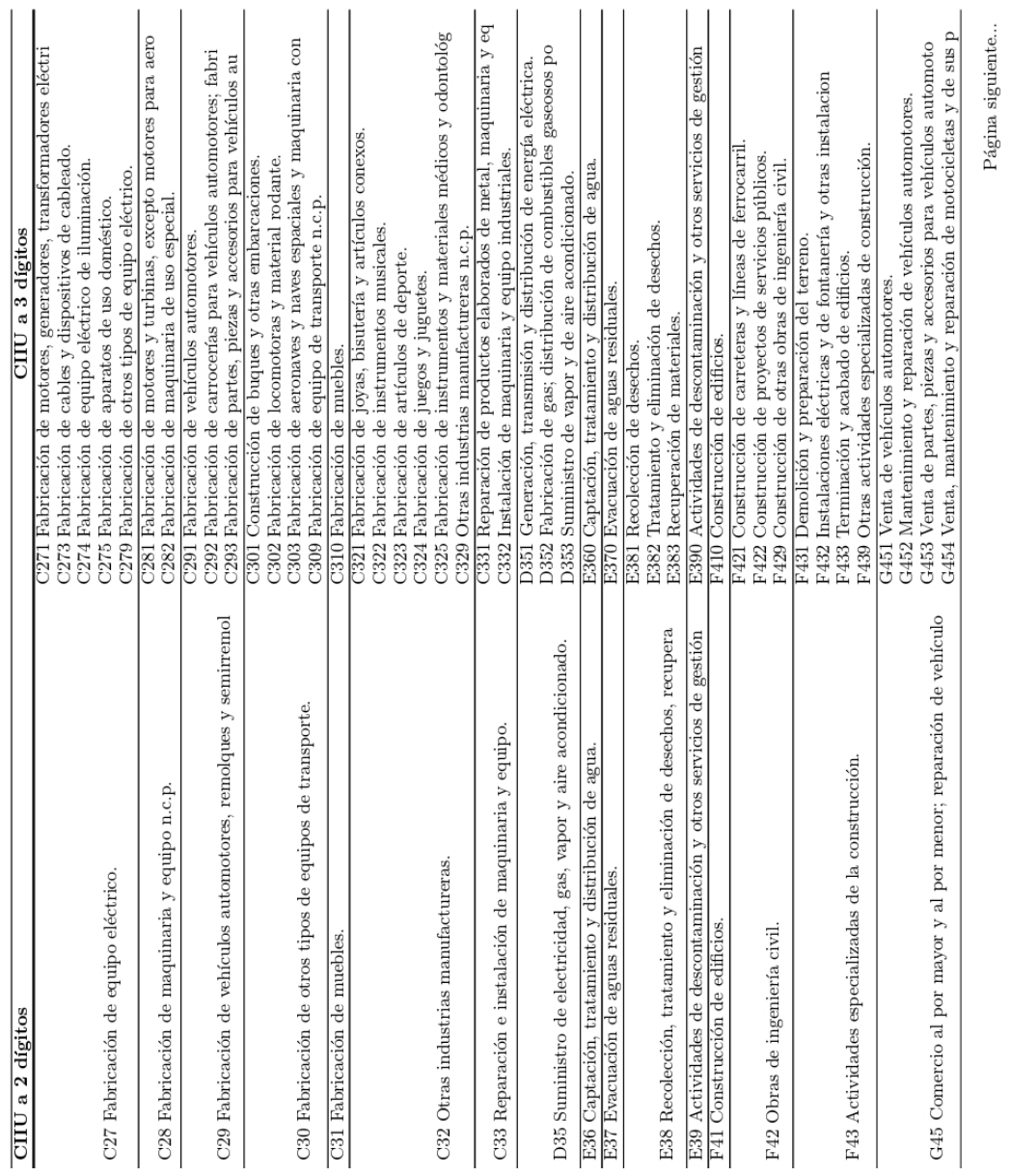




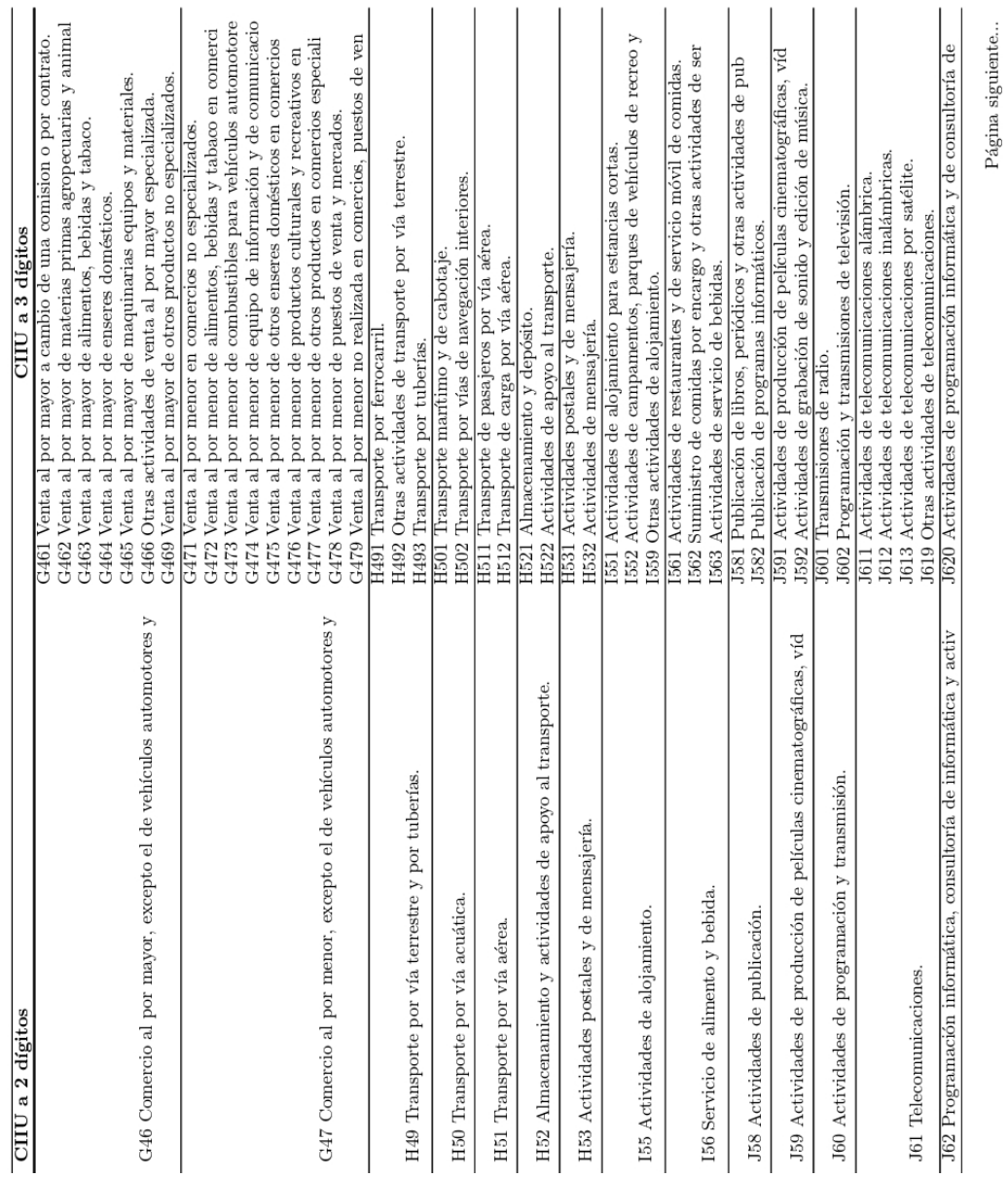




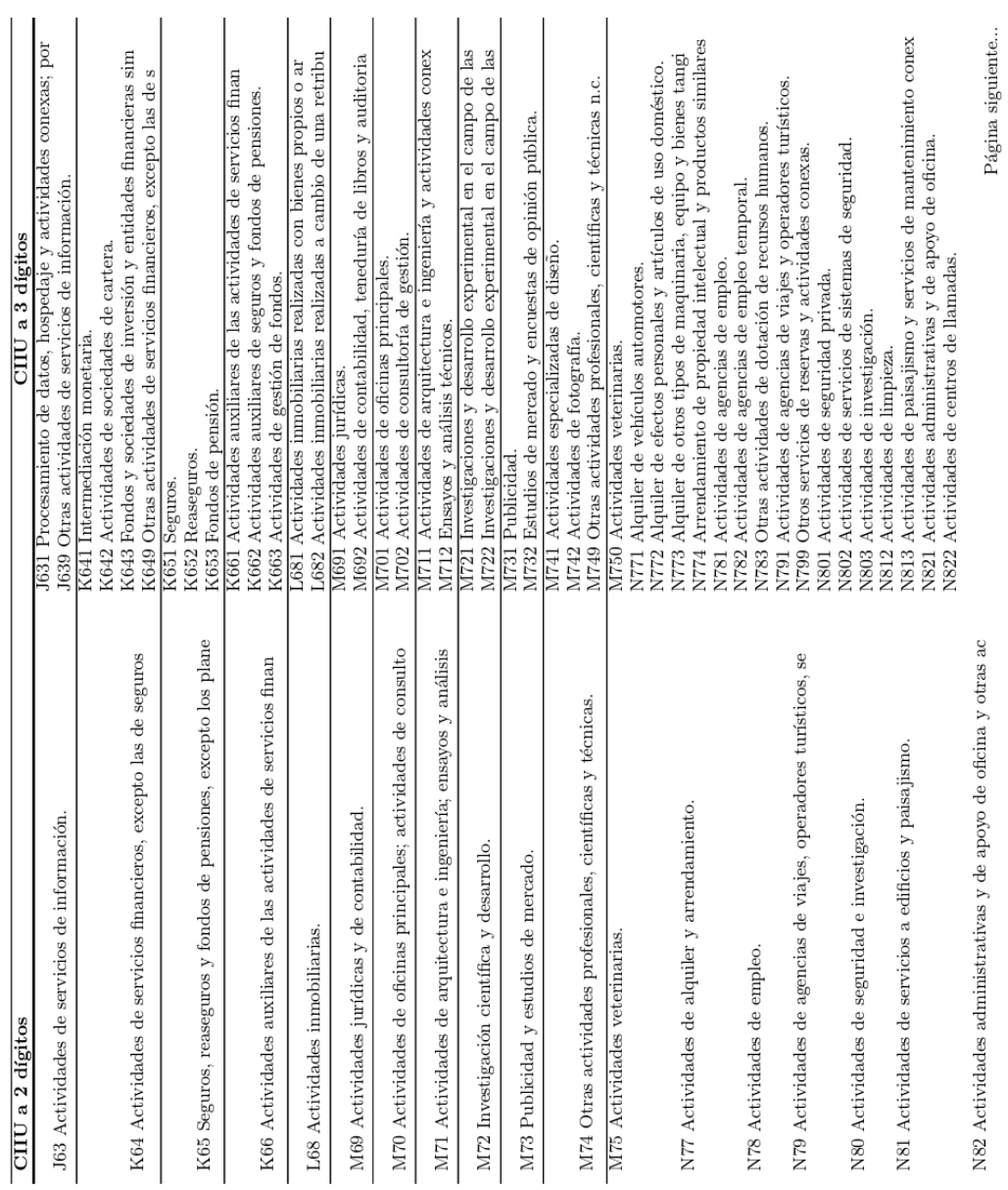




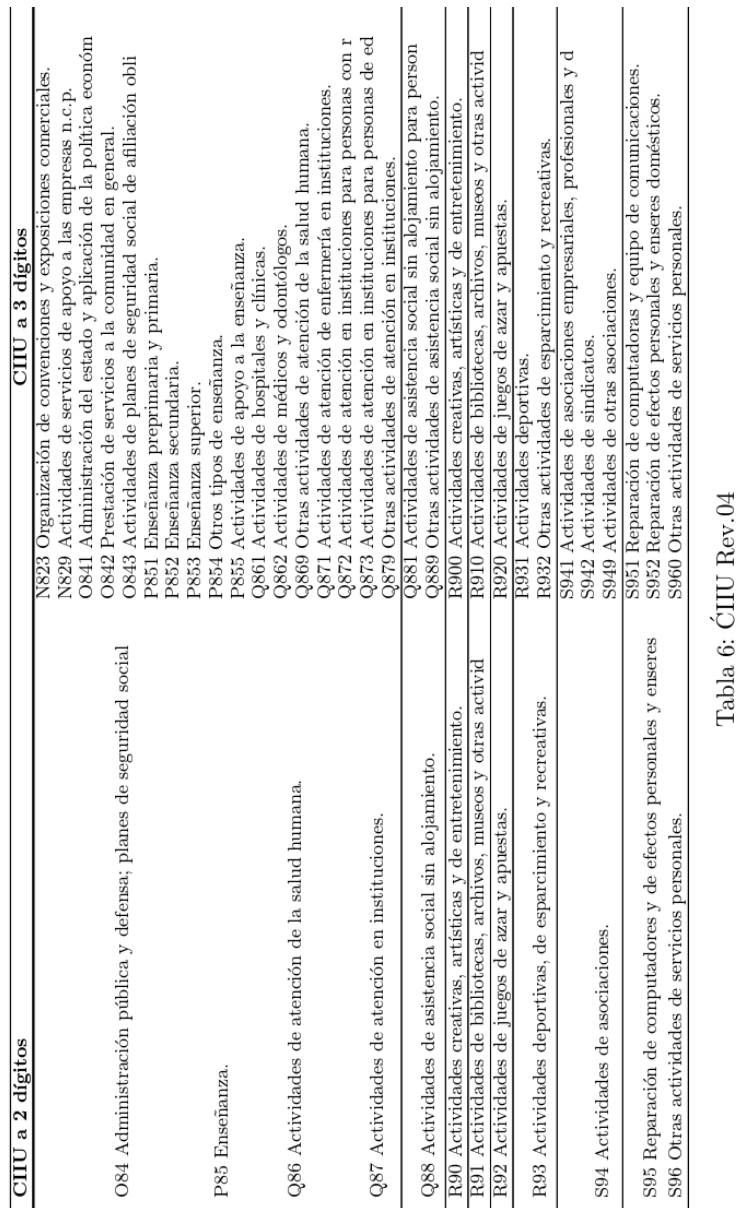




\section{B. Sin AGRUPACIÓN}

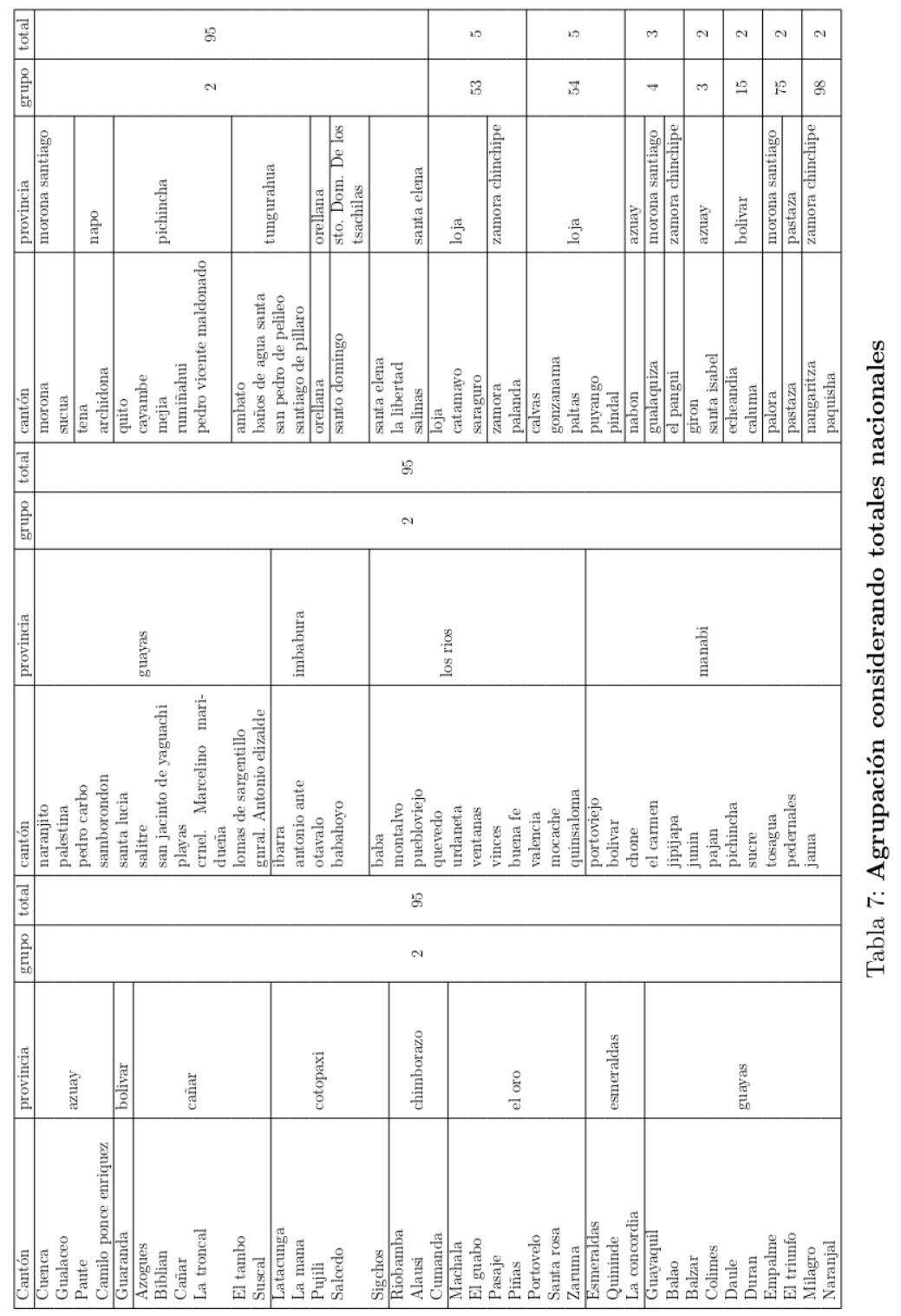




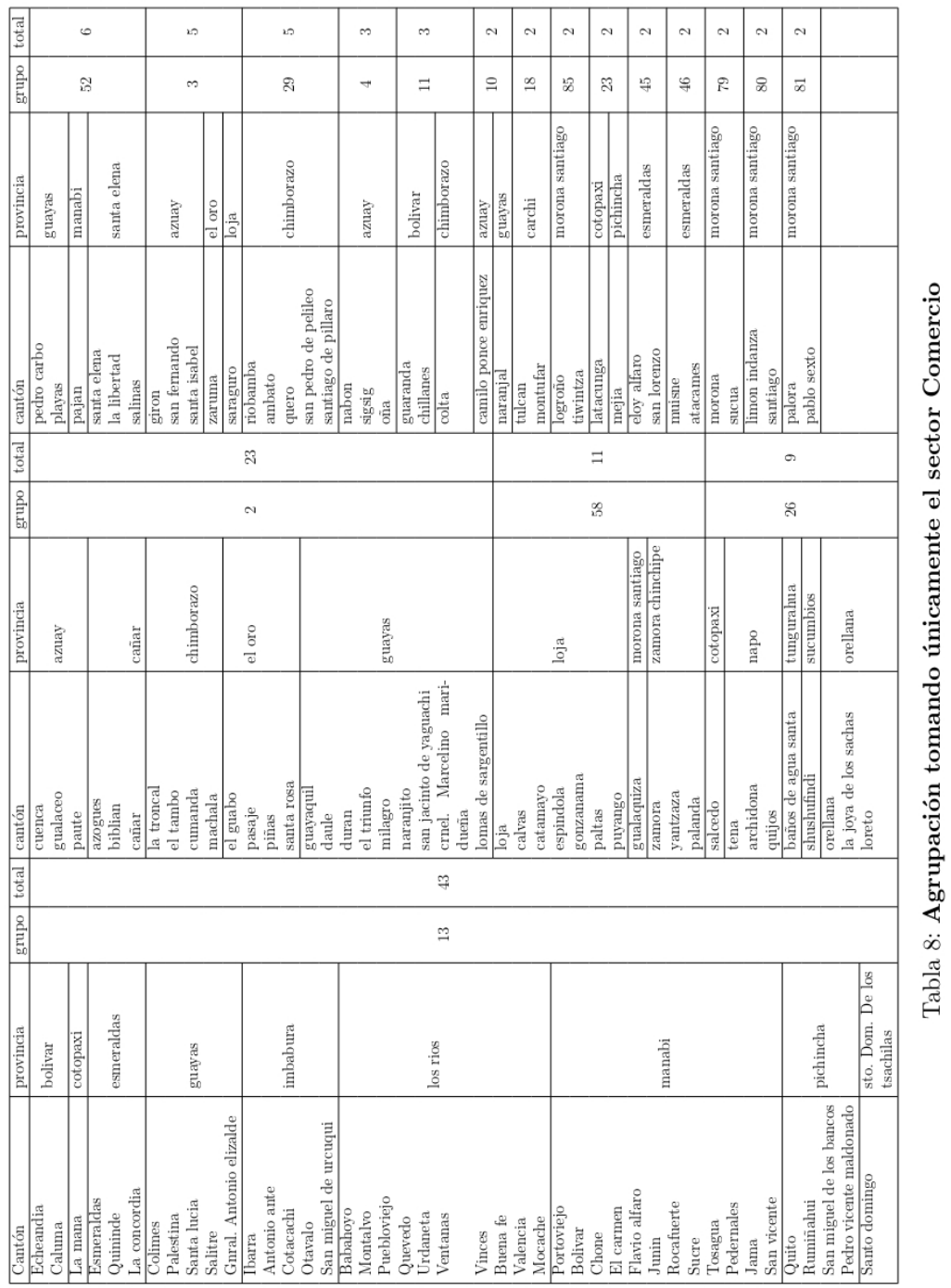




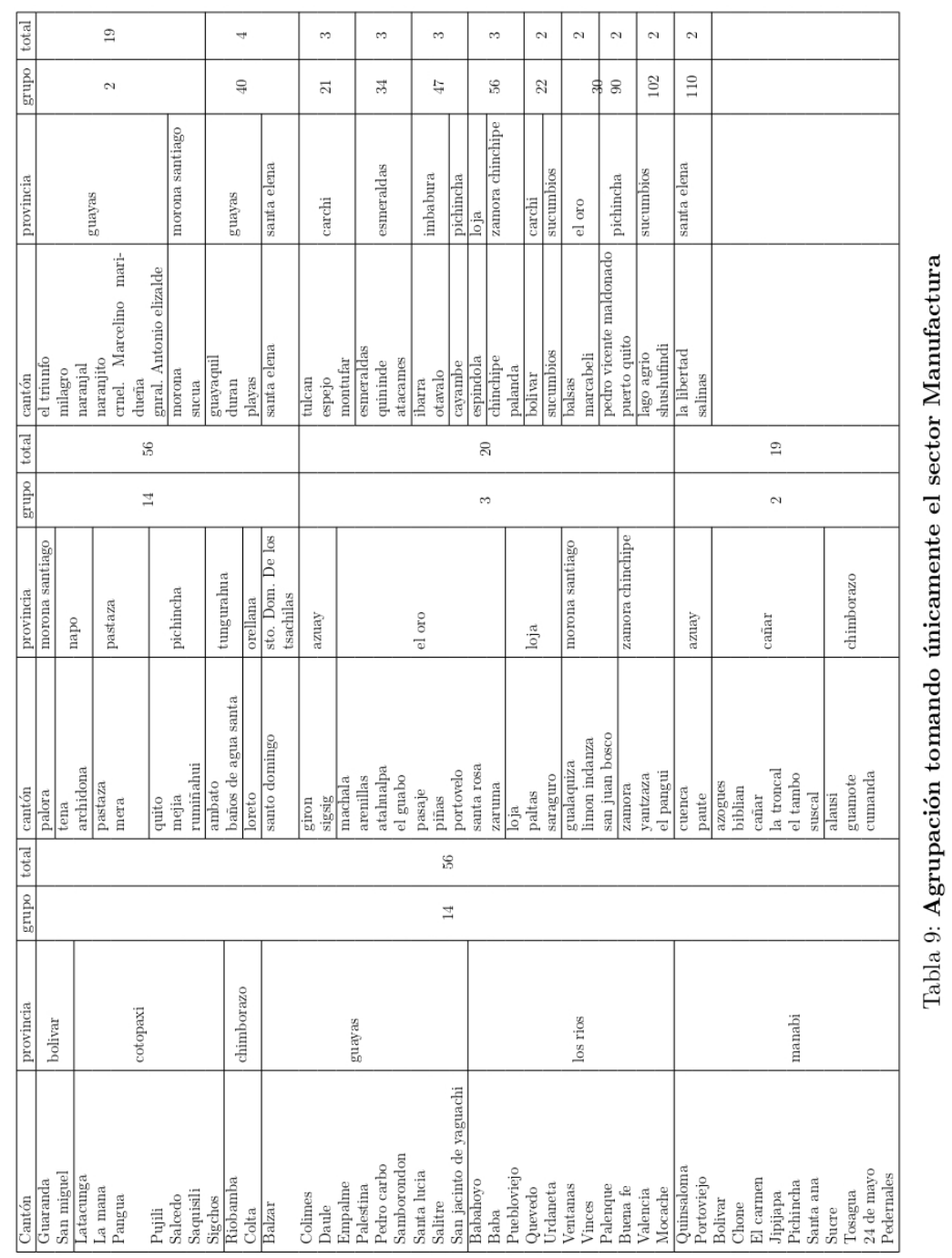




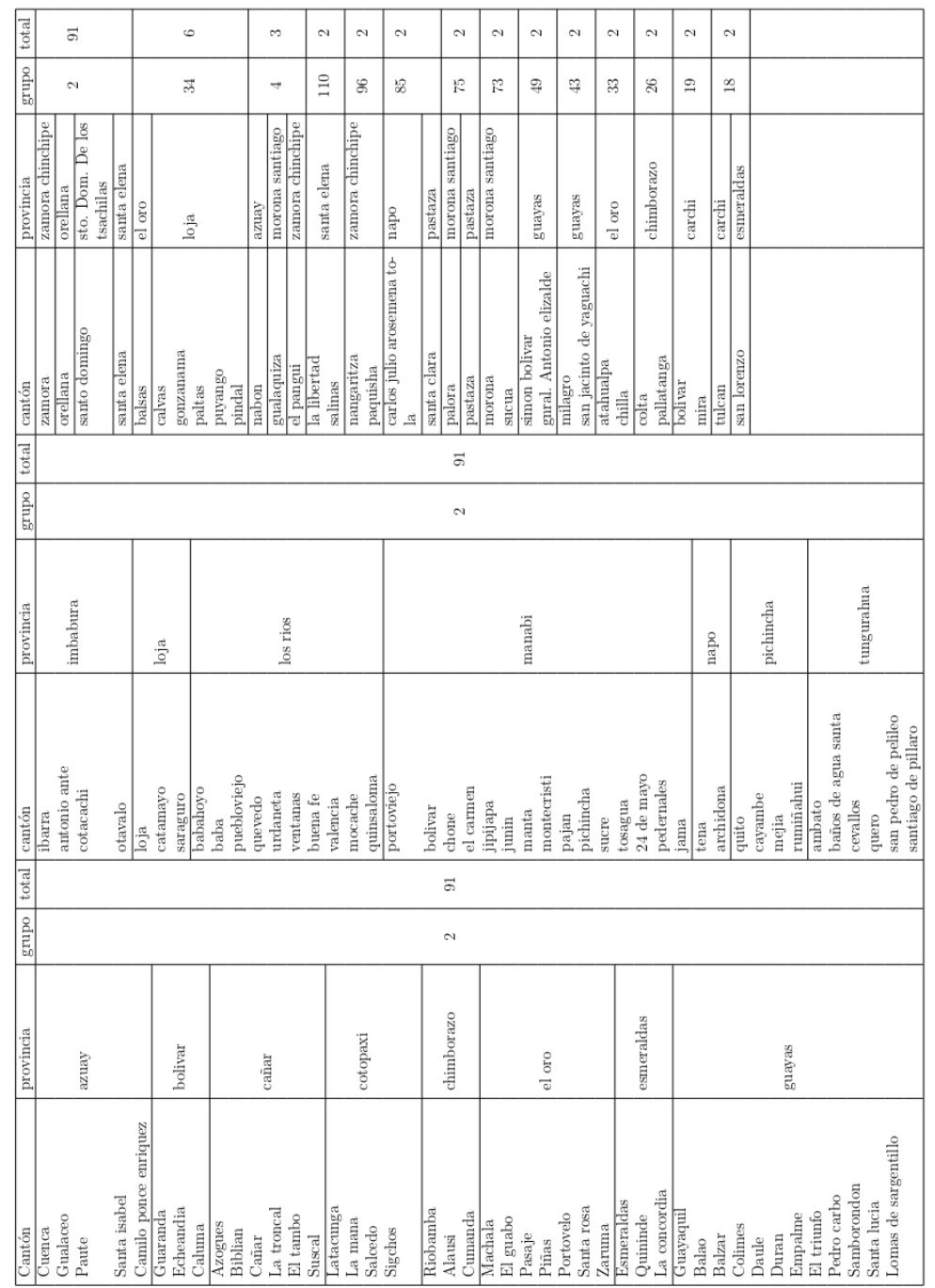

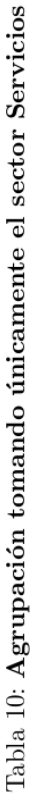




\section{C. Índices de concentración/especialización}

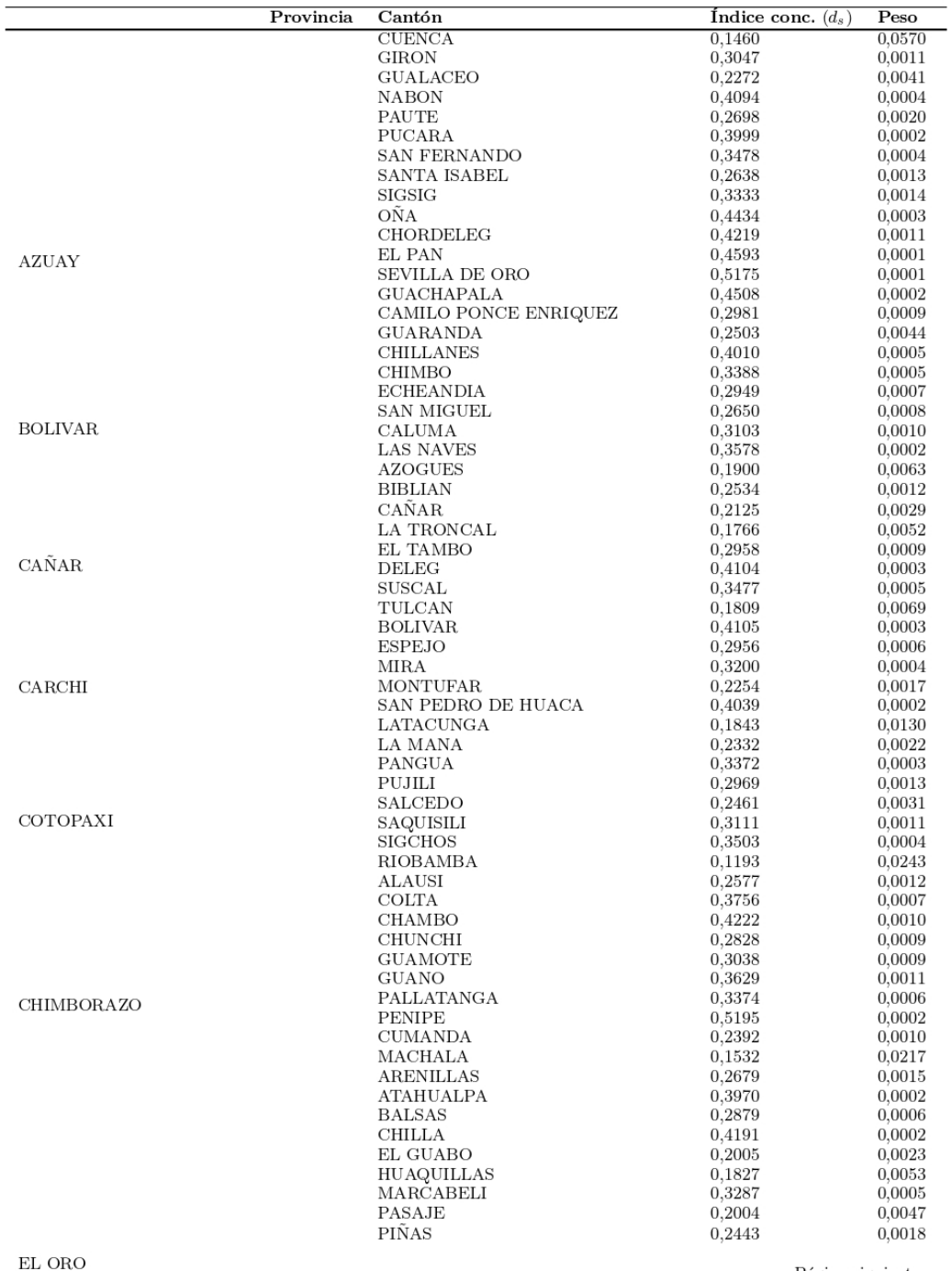




\begin{tabular}{|c|c|c|c|c|}
\hline & Provincia & Cantón & Índice conc. $\left(d_{s}\right)$ & Peso \\
\hline \multirow{31}{*}{ ESMERALDAS } & & PORTOVELO & 0,3028 & 0,0009 \\
\hline & & SANTA ROSA & 0,2039 & 0,0047 \\
\hline & & ZARUMA & 0,2683 & 0,0012 \\
\hline & & LAS LAJAS & 0,4244 & 0,0002 \\
\hline & & ESMERALDAS & 0,1368 & 0,0116 \\
\hline & & ELOY ALFARO & 0,3471 & 0,0009 \\
\hline & & MUISNE & 0,3603 & 0,0008 \\
\hline & & QUININDE & 0,2256 & 0,0040 \\
\hline & & SAN LORENZO & 0,3230 & 0,0010 \\
\hline & & ATACAMES & 0,3298 & 0,0027 \\
\hline & & RIOVERDE & 0,3912 & 0,0005 \\
\hline & & LA CONCORDIA & 0,2441 & 0,0030 \\
\hline & & GUAYAQUIL & 0,0950 & 0,1746 \\
\hline & & ALFREDO BAQUERIZO MORENO & 0,3399 & 0,0008 \\
\hline & & BALAO & 0,2747 & 0,0007 \\
\hline & & BALZAR & 0,2411 & 0,0024 \\
\hline & & COLIMES & 0,3418 & 0,0006 \\
\hline & & DAULE & 0,1946 & 0,0042 \\
\hline & & DURAN & 0,2076 & 0,0137 \\
\hline & & EMPALME & 0,2691 & 0,0030 \\
\hline & & EL TRIUNFO & 0,1942 & 0,0029 \\
\hline & & MILAGRO & 0,1720 & 0,0099 \\
\hline & & NARANJAL & 0,2404 & 0,0029 \\
\hline & & NARANJITO & 0,2705 & 0,0021 \\
\hline & & PALESTINA & 0,3786 & 0,0008 \\
\hline & & PEDRO CARBO & 0,2696 & 0,0022 \\
\hline & & SAMBORONDON & 0,2473 & 0,0029 \\
\hline & & SANTA LUCIA & 0,3165 & 0,0010 \\
\hline & & SALITRE & 0,3019 & 0,0011 \\
\hline & & SAN JACINTO DE YAGUACHI & 0,2488 & 0,0020 \\
\hline & & PLAYAS & 0,2633 & 0,0030 \\
\hline \multirow{10}{*}{ GUAYAS } & & SIMON BOLIVAR & 0,3538 & 0,0003 \\
\hline & & CRNEL. MARCELINO MARIDUEÑA & 0,2839 & 0,0006 \\
\hline & & LOMAS DE SARGENTILLO & 0,3476 & 0,0010 \\
\hline & & NOBOL & 0,3606 & 0,0008 \\
\hline & & GNRAL. ANTONIO ELIZALDE & 0,3230 & 0,0009 \\
\hline & & ISIDRO AYORA & 0,4074 & 0,0004 \\
\hline & & IBARRA & 0,1546 & 0,0190 \\
\hline & & ANTONIO ANTE & 0,2443 & 0,0032 \\
\hline & & COTACACHI & 0,2640 & 0,0019 \\
\hline & & OTAVALO & 0,1914 & 0,0076 \\
\hline \multirow[t]{14}{*}{ IMBABURA } & & PIMAMPIRO & 0,2923 & 0,0005 \\
\hline & & SAN MIGUEL DE URCUQUI & 0,3403 & 0,0004 \\
\hline & & LOJA & 0,1476 & 0,0237 \\
\hline & & CALVAS & 0,2357 & 0,0025 \\
\hline & & CATAMAYO & 0,2729 & 0,0034 \\
\hline & & CELICA & 0,3026 & 0,0008 \\
\hline & & CHAGUARPAMBA & 0,4078 & 0,0004 \\
\hline & & ESPINDOLA & 0,3244 & 0,0004 \\
\hline & & GONZANAMA & 0,3095 & 0,0005 \\
\hline & & MACARA & 0,2286 & 0,0021 \\
\hline & & PALTAS & 0,2971 & 0,0013 \\
\hline & & PUYANGO & 0,2633 & 0,0011 \\
\hline & & SARAGURO & 0,2888 & 0,0011 \\
\hline & & SOZORANGA & 0,4062 & 0,0001 \\
\hline \multirow{12}{*}{ LOJA } & & ZAPOTILLO & 0,3259 & 0,0005 \\
\hline & & PINDAL & 0,3296 & 0,0005 \\
\hline & & QUILANGA & 0,4400 & 0,0002 \\
\hline & & OLMEDO & 0,5635 & 0,0001 \\
\hline & & BABAHOYO & 0,2186 & 0,0081 \\
\hline & & $\mathrm{BABA}$ & 0,3013 & 0,0007 \\
\hline & & MONTALVO & 0,3005 & 0,0013 \\
\hline & & PUEBLOVIEJO & 0,2872 & 0,0011 \\
\hline & & QUEVEDO & 0,1968 & 0,0113 \\
\hline & & URDANETA & 0,3100 & 0,0011 \\
\hline & & VENTANAS & 0,2454 & 0,0033 \\
\hline & & VINCES & 0,2706 & 0,0022 \\
\hline
\end{tabular}

LOS RIOS 


\begin{tabular}{|c|c|c|c|c|}
\hline & Provincia & Cantón & Índice conc. $\left(d_{s}\right)$ & Peso \\
\hline & & PALENQUE & 0,3853 & 0,0004 \\
\hline & & BUENA FE & 0,2581 & 0,0032 \\
\hline & & VALENCIA & 0,2750 & 0,0012 \\
\hline & & MOCACHE & 0,3179 & 0,0010 \\
\hline & & QUINSALOMA & 0,3503 & 0,0007 \\
\hline & & PORTOVIEJO & 0,1773 & 0,0165 \\
\hline & & BOLIVAR & 0,2741 & 0,0013 \\
\hline & & CHONE & 0,2399 & 0,0039 \\
\hline & & EL CARMEN & 0,2012 & 0,0037 \\
\hline & & FLAVIO ALFARO & 0,3322 & 0,0006 \\
\hline & & JIPIJAPA & 0,2034 & 0,0033 \\
\hline & & JUNIN & 0,3449 & 0,0004 \\
\hline & & MANTA & 0,1238 & 0,0182 \\
\hline & & MONTECRISTI & 0,2956 & 0,0032 \\
\hline & & PAJAN & 0,2794 & 0,0009 \\
\hline & & PICHINCHA & 0,3541 & 0,0006 \\
\hline & & ROCAFUERTE & 0,2953 & 0,0013 \\
\hline & & SANTA ANA & 0,2906 & 0,0013 \\
\hline & & SUCRE & 0,2554 & 0,0023 \\
\hline & & TOSAGUA & 0,2650 & 0,0012 \\
\hline & & 24 DE MAYO & 0,3266 & 0,0005 \\
\hline & & PEDERNALES & 0,2768 & 0,0020 \\
\hline \multirow[t]{14}{*}{ MANABI } & & OLMEDO & 0,3706 & 0,0003 \\
\hline & & PUERTO LOPEZ & 0,3341 & 0,0015 \\
\hline & & JAMA & 0,3521 & 0,0004 \\
\hline & & JARAMIJO & 0,3603 & 0,0008 \\
\hline & & SAN VICENTE & 0,3007 & 0,0008 \\
\hline & & MORONA & 0,2132 & 0,0040 \\
\hline & & GUALAQUIZA & 0,2406 & 0,0012 \\
\hline & & LIMON INDANZA & 0,3269 & 0,0006 \\
\hline & & PALORA & 0,3512 & 0,0005 \\
\hline & & SANTIAGO & 0,3408 & 0,0005 \\
\hline & & SUCUA & 0,2479 & 0,0014 \\
\hline & & HUAMBOYA & 0,4176 & 0,0002 \\
\hline & & SAN JUAN BOSCO & 0,3736 & 0,0002 \\
\hline & & TAISHA & 0,4879 & 0,0001 \\
\hline \multirow[t]{6}{*}{ MORONA SANTIAGO } & & LOGROÑO & 0,4309 & 0,0002 \\
\hline & & PABLO SEXTO & 0,4980 & 0,0001 \\
\hline & & $\begin{array}{l}\text { TIWINTZA } \\
\text { TIU }\end{array}$ & 0,4617 & 0,0002 \\
\hline & & TENA & 0,1970 & 0,0037 \\
\hline & & ARCHIDONA & 0,3210 & 0,0007 \\
\hline & & EL CHACO & 0,3442 & 0,0005 \\
\hline \multirow{4}{*}{ NAPO } & & QUIJOS & 0,4217 & 0,0002 \\
\hline & & CARLOS JULIO AROSEMENA TOLA & 0,4123 & 0,0001 \\
\hline & & PASTAZA & 0,1730 & 0,0060 \\
\hline & & MERA & 0,3435 & 0,0009 \\
\hline \multirow{7}{*}{ PASTAZA } & & SANTA CLARA & 0,4549 & 0,0001 \\
\hline & & $\begin{array}{l}\text { ARAJUNO } \\
\text { ARA }\end{array}$ & 0,5149 & 0,0001 \\
\hline & & QUITO & 0,1056 & 0,2020 \\
\hline & & CAYAMBE & 0,2140 & 0,0055 \\
\hline & & MEJIA & 0,1835 & 0,0050 \\
\hline & & PEDRO MONCAYO & 0,3123 & 0,0012 \\
\hline & & RUMIÑAHUI & 0,1653 & 0,0087 \\
\hline \multirow{9}{*}{ PICHINCHA } & & SAN MIGUEL DE LOS BANCOS & 0,3491 & 0,0007 \\
\hline & & PEDRO VICENTE MALDONADO & 0,2602 & 0,0010 \\
\hline & & PUERTO QUITO & 0,3248 & 0,0007 \\
\hline & & AMBATO & 0,1462 & 0,0370 \\
\hline & & BAÑOS DE AGUA SANTA & 0,2975 & 0,0030 \\
\hline & & CEVALLOS & 0,3619 & 0,0005 \\
\hline & & MOCHA & 0,4184 & 0,0004 \\
\hline & & PATATE & 0,3285 & 0,0006 \\
\hline & & QUERO & 0,3016 & 0,0007 \\
\hline \multirow{5}{*}{ TUNGURAHUA } & & SAN PEDRO DE PELILEO & 0,2789 & 0,0037 \\
\hline & & SANTIAGO DE PILLARO & 0,2505 & 0,0020 \\
\hline & & TISALEO & 0,4056 & 0,0003 \\
\hline & & ZAMORA & 0,2493 & 0,0022 \\
\hline & & CHINCHIPE & 0,3070 & 0,0006 \\
\hline
\end{tabular}




\begin{tabular}{|c|c|c|c|}
\hline Provincia & Cantón & Índice conc. $\left(d_{s}\right)$ & Peso \\
\hline \multirow{16}{*}{ SUCUMBIOS } & NANGARITZA & 0,3852 & 0,0003 \\
\hline & YACUAMBI & 0,4700 & 0,0002 \\
\hline & YANTZAZA & 0,2422 & 0,0017 \\
\hline & EL PANGUI & 0,3106 & 0,0006 \\
\hline & CENTINELA DEL CONDOR & 0,3841 & 0,0004 \\
\hline & PALANDA & 0,3092 & 0,0004 \\
\hline & PAQUISHA & 0,4300 & 0,0002 \\
\hline & LAGO AGRIO & 0,1734 & 0,0065 \\
\hline & GONZALO PIZARRO & 0,4345 & 0,0002 \\
\hline & PUTUMAYO & 0,4855 & 0,0002 \\
\hline & SHUSHUFINDI & 0,2721 & 0,0017 \\
\hline & SUCUMBIOS & 0,5646 & 0,0001 \\
\hline & CASCALES & 0,3734 & 0,0003 \\
\hline & CUYABENO & 0,3645 & 0,0002 \\
\hline & ORELLANA & 0,2035 & 0,0045 \\
\hline & AGUARICO & 0,7362 & 0,00003 \\
\hline \multirow{2}{*}{ ORELLANA } & LA JOYA DE LOS SACHAS & 0,2585 & 0,0012 \\
\hline & LORETO & 0,3068 & 0,0006 \\
\hline \multirow[t]{2}{*}{ SANTO DOMINGO DE LOS TSACHILAS } & SANTO DOMINGO & 0,1303 & 0,0292 \\
\hline & SANTA ELENA & 0,2534 & 0,0046 \\
\hline \multirow[b]{2}{*}{ SANTA ELENA } & LA LIBERTAD & 0,1911 & 0,0092 \\
\hline & SALINAS & 0,2752 & 0,0040 \\
\hline
\end{tabular}

Tabla 11: Índice de concentración económica con relación a la media nacional $(0.1635)$

\begin{tabular}{|c|c|c|c|}
\hline Sector económico & Actividad económica & Índice (esp.) & Peso \\
\hline & C101 Elaboración y conservación de carne. & 0,5252 & 0,0003 \\
\hline & C102 Elaboración y conservación de pescados, crustáceos y molusco & 0,8081 & 0,0000 \\
\hline & C103 Elaboración y conservación de frutas, legumbres y hortalizas & 0,4786 & 0,0004 \\
\hline & C104 Elaboración de aceites y grasas de origen vegetal y animal. & 0,7224 & 0,0001 \\
\hline & C105 Elaboración de productos lácteos. & 0,4402 & 0,0009 \\
\hline & C106 Elaboración de productos de molinería, almidones y productos & 0,5776 & 0,0007 \\
\hline & C107 Elaboración de otros productos alimenticios. & 0,0972 & 0,0172 \\
\hline & C108 Elaboración de alimentos preparados para animales. & 0,6628 & 0,0001 \\
\hline & C110 Elaboración de bebidas. & 0,4424 & 0,0004 \\
\hline & C120 Elaboración de productos de tabaco. & 0,6620 & 0,0000 \\
\hline & C131 Hilatura, tejedura y acabados de productos textiles. & 0,4214 & 0,0005 \\
\hline & C139 Fabricación de otros productos textiles. & 0,3365 & 0,0025 \\
\hline & C141 Fabricación de prendas de vestir, excepto prendas de piel. & 0,1689 & 0,0168 \\
\hline & C142 Fabricación de artículos de piel. & 0,6920 & 0,0000 \\
\hline & C143 Fabricación de artículos de punto y ganchillo. & 0,6685 & 0,0002 \\
\hline & C151 Curtido y adobo de cueros; fabricación de maletas, bolsos de & 0,5029 & 0,0007 \\
\hline & C152 Fabricación de calzado. & 0,4706 & 0,0020 \\
\hline & C161 Aserrado y acepilladura de madera. & 0,4980 & 0,0005 \\
\hline & C162 Fabricación de hojas de madera para enchapado y tableros a b & 0,2474 & 0,0058 \\
\hline & C170 Fabricación de papel y de productos de papel. & 0,4536 & 0,0005 \\
\hline & C181 Impresión y actividades de servicios relacionados con la imp & 0,2760 & 0,0041 \\
\hline & C182 Reproducción de grabaciones. & 0,7092 & 0,0000 \\
\hline & C192 Fabricación de productos de la refinación del petróleo. & 0,6386 & 0,0000 \\
\hline & C201 Fabricación de sustancias químicas básicas, de abonos y comp & 0,4753 & 0,0002 \\
\hline & C202 Fabricación de otros productos químicos. & 0,4240 & 0,0004 \\
\hline & C203 Fabricación de fibras artificiales. & 0,5985 & 0,0000 \\
\hline & C210 Fabricación de productos farmacéuticos, sustancias químicas & 0,5207 & 0,0001 \\
\hline & C221 Fabricación de productos de caucho. & 0,4059 & 0,0003 \\
\hline & C222 Fabricación de productos de plástico. & 0,4179 & 0,0007 \\
\hline & C231 Fabricación de vidrio y productos de vidrio. & 0,4113 & 0,0004 \\
\hline & C239 Fabricación de productos minerales no metálicos n.c.p. & 0,3702 & 0,0049 \\
\hline & C241 Industrias básicas de hierro y acero. & 0,5293 & 0,0002 \\
\hline & C242 Fabricación de productos primarios de metales preciosos y me & 0,5425 & 0,0001 \\
\hline & C243 Fundición de metales. & 0,5533 & 0,0001 \\
\hline & C251 Fabricación de productos metálicos para uso estructural, tan & 0,1100 & 0,0127 \\
\hline & C252 Fabricación de armas y municiones. & 0,7790 & 0,0000 \\
\hline
\end{tabular}




\begin{tabular}{|c|c|c|c|}
\hline Sector económico & Actividad económica & Índice (esp.) & Peso \\
\hline & C259 Fabricación de otros productos elaborados de metal, activida & 0,2334 & 0,0035 \\
\hline & C261 Fabricación de componentes y tableros electrónicos. & 0,6206 & 0,0000 \\
\hline & C262 Fabricación de ordenadores y equipo periférico. & 0,7420 & 0,0000 \\
\hline & C263 Fabricación de equipo de comunicaciones. & 0,6411 & 0,0000 \\
\hline & C264 Fabricación de aparatos electrónicos de consumo. & 0,7384 & 0,0000 \\
\hline & C265 Fabricación de equipos de medición, prueba, navegación, cont & 0,6680 & 0,0000 \\
\hline & C266 Fabricación de equipo de irradiación, y equipo electrónico d & 0,6468 & 0,0000 \\
\hline & C267 Fabricación de instrumentos ópticos y equipo fotográficos. & 0,5992 & 0,0000 \\
\hline & C271 Fabricación de motores, generadores, transformadores eléctri & 0,5881 & 0,0000 \\
\hline & C273 Fabricación de cables y dispositivos de cableado. & 0,6474 & 0,0000 \\
\hline & C274 Fabricación de equipo eléctrico de iluminación. & 0,5833 & 0,0000 \\
\hline & C275 Fabricación de aparatos de uso doméstico. & 0,5149 & 0,0002 \\
\hline & C279 Fabricación de otros tipos de equipo eléctrico. & 0,6818 & 0,0000 \\
\hline & C281 Fabricación de motores y turbinas, excepto motores para aero & 0,4216 & 0,0005 \\
\hline & C282 Fabricación de maquinaria de uso especial. & 0,4077 & 0,0004 \\
\hline & C291 Fabricación de vehículos automotores. & 0,6469 & 0,0000 \\
\hline & C292 Fabricación de carrocerías para vehículos automotores; fabri & 0,4945 & 0,0004 \\
\hline & C293 Fabricación de partes, piezas y accesorios para vehículos au & 0,3960 & 0,0004 \\
\hline & C301 Construcción de buques y otras embarcaciones. & 0,7431 & 0,0000 \\
\hline & C302 Fabricación de locomotoras y material rodante. & 0,7255 & 0,0000 \\
\hline & C303 Fabricación de aeronaves y naves espaciales y maquinaria con & 0,8133 & 0,0000 \\
\hline & C309 Fabricación de equipo de transporte n.c.p. & 0,5654 & 0,0001 \\
\hline & C310 Fabricación de muebles. & 0,1551 & 0,0117 \\
\hline & C321 Fabricación de joyas, bisutería y artículos conexos. & 0,4104 & 0,0012 \\
\hline & C322 Fabricación de instrumentos musicales. & 0,5698 & 0,0002 \\
\hline & C323 Fabricación de artículos de deporte. & 0,6107 & 0,0000 \\
\hline & C324 Fabricación de juegos y juguetes. & 0,4504 & 0,0002 \\
\hline & C325 Fabricación de instrumentos y materiales médicos y odontológ & 0,4124 & 0,0004 \\
\hline & C329 Otras industrias manufactureras n.c.p. & 0,4030 & 0,0011 \\
\hline & C331 Reparación de productos elaborados de metal, maquinaria y eq & 0,2476 & 0,0027 \\
\hline & C332 Instalación de maquinaria y equipo industriales. & 0,5464 & 0,0001 \\
\hline \multirow{17}{*}{ Servicios } & D351 Generación, transmisión y distribución de energía eléctrica. & 0,5382 & 0,0004 \\
\hline & D352 Fabricación de gas; distribución de combustibles gaseosos po & 0,8650 & 0,0000 \\
\hline & D353 Suministro de vapor y de aire acondicionado. & 0,6400 & 0,0001 \\
\hline & E360 Captación, tratamiento y distribución de agua. & 0,5303 & 0,0003 \\
\hline & E370 Evacuación de aguas residuales. & 0,7435 & 0,0000 \\
\hline & E381 Recolección de desechos. & 0,5186 & 0,0002 \\
\hline & E382 Tratamiento y eliminación de desechos. & 0,8214 & 0,0000 \\
\hline & E383 Recuperación de materiales. & 0,6104 & 0,0001 \\
\hline & E390 Actividades de descontaminación y otros servicios de gestión & 0,7932 & 0,0000 \\
\hline & F410 Construcción de edificios. & 0,3687 & 0,0010 \\
\hline & F421 Construcción de carreteras y líneas de ferrocarril. & 0,4945 & 0,0003 \\
\hline & F422 Construcción de proyectos de servicios públicos. & 0,5067 & 0,0002 \\
\hline & F429 Construcción de otras obras de ingeniería civil. & 0,5723 & 0,0001 \\
\hline & F431 Demolición y preparación del terreno. & 0,6322 & 0,0000 \\
\hline & F432 Instalaciones eléctricas y de fontanería y otras instalacion & 0,4051 & 0,0006 \\
\hline & F433 Terminación y acabado de edificios. & 0,4416 & 0,0007 \\
\hline & F439 Otras actividades especializadas de construcción. & 0,4695 & 0,0002 \\
\hline \multirow{19}{*}{ Comercio } & G451 Venta de vehículos automotores. & 0,3633 & 0,0015 \\
\hline & G452 Mantenimiento y reparación de vehículos automotores. & 0,0973 & 0,0417 \\
\hline & G453 Venta de partes, piezas y accesorios para vehículos automoto & 0,1726 & 0,0107 \\
\hline & G454 Venta, mantenimiento y reparación de motocicletas y de sus $\mathrm{p}$ & 0,3153 & 0,0038 \\
\hline & G461 Venta al por mayor a cambio de una comision o por contrato. & 0,3361 & 0,0017 \\
\hline & G462 Venta al por mayor de materias primas agropecuarias y animal & 0,4060 & 0,0008 \\
\hline & G463 Venta al por mayor de alimentos, bebidas y tabaco. & 0,2839 & 0,0035 \\
\hline & G464 Venta al por mayor de enseres domésticos. & 0,3285 & 0,0030 \\
\hline & G465 Venta al por mayor de maquinarias equipos y materiales. & 0,3204 & 0,0017 \\
\hline & G466 Otras actividades de venta al por mayor especializada. & 0,2160 & 0,0034 \\
\hline & G469 Venta al por mayor de otros productos no especializados. & 0,4832 & 0,0002 \\
\hline & G471 Venta al por menor en comercios no especializados. & 0,0931 & 0,2074 \\
\hline & G472 Venta al por menor de alimentos, bebidas y tabaco en comerci & 0,1509 & 0,0433 \\
\hline & G473 Venta al por menor de combustibles para vehículos automotore & 0,1849 & 0,0038 \\
\hline & G474 Venta al por menor de equipo de información y de comunicacio & 0,1755 & 0,0078 \\
\hline & G475 Venta al por menor de otros enseres domésticos en comercios & 0,0645 & 0,0380 \\
\hline & G476 Venta al por menor de productos culturales y recreativos en & 0,1437 & 0,0251 \\
\hline & G477 Venta al por menor de otros productos en comercios especiali & 0,0657 & 0,0935 \\
\hline & G478 Venta al por menor de puestos de venta y mercados. & 0,3471 & 0,0461 \\
\hline
\end{tabular}




\begin{tabular}{|c|c|c|c|}
\hline Sector económico & Actividad económica & Índice (esp.) & Peso \\
\hline & G479 Venta al por menor no realizada en comercios, puestos de ven & 0,4130 & 0,0009 \\
\hline & H491 Transporte por ferrocarril. & 0,6604 & 0,0000 \\
\hline & H492 Otras actividades de transporte por vía terrestre. & 0,1755 & 0,0058 \\
\hline & H493 Transporte por tuberías. & 0.7481 & 0,0000 \\
\hline & H501 Transporte marítimo y de cabotaje. & 0,5824 & 0,0001 \\
\hline & H502 Transporte por vías de navegación interiores. & 0,7253 & 0,0000 \\
\hline & H511 Transporte de pasajeros por vía aérea. & 0,5768 & 0,0001 \\
\hline & H512 Transporte de carga por vía aérea. & 0,5623 & 0,0001 \\
\hline & H521 Almacenamiento y depósito. & 0,5470 & 0,0002 \\
\hline & H522 Actividades de apoyo al transporte. & 0,3362 & 0,0029 \\
\hline & H531 Actividades postales y de mensajería. & 0,4101 & 0,0004 \\
\hline & H532 Actividades de mensajería. & 0,3422 & 0,0010 \\
\hline & I551 Actividades de alojamiento para estancias cortas. & 0,2649 & 0,0070 \\
\hline & I552 Actividades de campamentos, parques de vehículos de recreo y & 0,7465 & 0,0000 \\
\hline & I559 Otras actividades de alojamiento. & 0,6241 & 0,0001 \\
\hline & I561 Actividades de restaurantes y de servicio móvil de comidas. & 0,0558 & 0,0912 \\
\hline & I562 Suministro de comidas por encargo y otras actividades de ser & 0,3910 & 0,0007 \\
\hline & I563 Actividades de servicio de bebidas. & 0.2827 & 0,0067 \\
\hline & J581 Publicación de libros, periódicos y otras actividades de pub & 0,3728 & 0,0009 \\
\hline & J582 Publicación de programas informáticos. & 0,6535 & 0,0001 \\
\hline & J591 Actividades de producción de películas cinematográficas, víd & 0,4723 & 0,0002 \\
\hline & J592 Actividades de grabación de sonido y edición de música. & 0,4840 & 0,0002 \\
\hline & J601 Transmisiones de radio. & 0,3338 & 0,0008 \\
\hline & J602 Programación y transmisiones de televisión. & 0,3863 & 0,0005 \\
\hline & J611 Actividades de telecomunicaciones alámbrica. & 0,4107 & 0,0008 \\
\hline & J612 Actividades de telecomunicaciones inalámbricas. & 0,4903 & 0.0004 \\
\hline & J613 Actividades de telecomunicaciones por satélite. & 0,5807 & 0,0001 \\
\hline & J619 Otras actividades de telecomunicaciones. & 0,0879 & 0,0352 \\
\hline & J620 Actividades de programación informática y de consultoría de & 0,4481 & 0,0006 \\
\hline & J631 Procesamiento de datos, hospedaje y actividades conexas; por & 0,5665 & 0,0001 \\
\hline & J639 Otras actividades de servicios de información. & 0,6885 & 0,0000 \\
\hline & K641 Intermediación monetaria. & 0.2288 & 0,0040 \\
\hline & K642 Actividades de sociedades de cartera. & 0,6507 & 0,0000 \\
\hline & K643 Fondos y sociedades de inversión y entidades financieras sim & 0,5593 & 0,0000 \\
\hline & K649 Otras actividades de servicios financieros, excepto las de $\mathrm{s}$ & 0,3718 & 0,0008 \\
\hline & K651 Seguros. & 0,3788 & 0,0009 \\
\hline & K652 Reaseguros. & 0,5533 & 0,0000 \\
\hline & K653 Fondos de pensión. & 0,6849 & 0,0000 \\
\hline & K661 Actividades auxiliares de las actividades de servicios finan & 0,5176 & 0,0003 \\
\hline & K662 Actividades auxiliares de seguros y fondos de pensiones. & 0,5402 & 0,0001 \\
\hline & K663 Actividades de gestión de fondos. & 0,6114 & 0,0000 \\
\hline & L681 Actividades inmobiliarias realizadas con bienes propios o ar & 0,3198 & 0,0018 \\
\hline & L682 Actividades inmobiliarias realizadas a cambio de una retribu & 0,3800 & 0,0016 \\
\hline & M691 Actividades jurídicas. & 0,2031 & 0,0132 \\
\hline & M692 Actividades de contabilidad, teneduría de libros y auditoria & 0,2423 & 0,0024 \\
\hline & M701 Actividades de oficinas principales. & 0,5460 & 0,0001 \\
\hline & M702 Actividades de consultoría de gestión. & 0,4087 & 0,0009 \\
\hline & M711 Actividades de arquitectura e ingeniería y actividades conex & 0,3601 & 0,0026 \\
\hline & M712 Ensayos y análisis técnicos. & 0,5398 & 0,0001 \\
\hline & M721 Investigaciones y desarrollo experimental en el campo de las & 0,4852 & 0,0002 \\
\hline & M722 Investigaciones y desarrollo experimental en el campo de las & 0,5875 & 0,0001 \\
\hline & M731 Publicidad. & 0,2689 & 0,0020 \\
\hline & M732 Estudios de mercado y encuestas de opinión pública. & 0,5377 & 0,0002 \\
\hline & M741 Actividades especializadas de diseño. & 0,3342 & 0,0009 \\
\hline & M742 Actividades de fotografía. & 0,1698 & 0,0025 \\
\hline & M749 Otras actividades profesionales, científicas y técnicas n.c. & 0,4433 & 0,0005 \\
\hline & M750 Actividades veterinarias. & 0,2960 & 0,0012 \\
\hline & N771 Alquiler de vehículos automotores. & 0,4636 & 0,0002 \\
\hline & N772 Alquiler de efectos personales y artículos de uso doméstico. & 0,2532 & 0,0017 \\
\hline & N773 Alquiler de otros tipos de maguinaria, equipo y bienes tangi & 0,3747 & 0,0007 \\
\hline & N774 Arrendamiento de propiedad intelectual y productos similares & 0.6114 & 0,0000 \\
\hline & N781 Actividades de agencias de empleo. & 0,5164 & 0,0001 \\
\hline & N782 Actividades de agencias de empleo temporal. & 0,6407 & 0,0000 \\
\hline & N783 Otras actividades de dotación de recursos humanos. & 0,6361 & 0,0000 \\
\hline & N791 Actividades de agencias de viajes y operadores turísticos. & 0,4184 & 0,0014 \\
\hline & N799 Otros servicios de reservas y actividades conexas. & 0,5720 & 0,0002 \\
\hline & N801 Actividades de seguridad privada. & 0,3898 & 0,0012 \\
\hline
\end{tabular}




\begin{tabular}{|c|c|c|c|}
\hline Sector económico & Actividad económica & Índice (esp.) & Peso \\
\hline & N802 Actividades de servicios de sistemas de seguridad. & 0,4608 & 0,0002 \\
\hline & N803 Actividades de investigación. & 0,8309 & 0,0000 \\
\hline & N812 Actividades de limpieza. & 0,4713 & 0,0005 \\
\hline & N813 Actividades de paisajismo y servicios de mantenimiento conex & 0,7042 & 0,0000 \\
\hline & N821 Actividades administrativas y de apoyo de oficina. & 0,2037 & 0,0048 \\
\hline & N822 Actividades de centros de llamadas. & 0,5703 & 0,0001 \\
\hline & N823 Organización de convenciones y exposiciones comerciales. & 0.4768 & 0,0002 \\
\hline & N829 Actividades de servicios de apoyo a las empresas n.c.p. & 0,4050 & 0,0006 \\
\hline & O841 Administración del estado y aplicación de la política económ & 0,3431 & 0,0040 \\
\hline & O842 Prestación de servicios a la comunidad en general. & 0,3137 & 0,0023 \\
\hline & O843 Actividades de planes de seguridad social de afiliación obli & 0,5887 & 0,0001 \\
\hline & P851 Enseñanza preprimaria y primaria. & 0,1285 & 0,0164 \\
\hline & P852 Enseñanza secundaria. & 0,1496 & 0,0078 \\
\hline & P853 Enseñanza superior. & 0,3229 & 0,0011 \\
\hline & P854 Otros tipos de enseñanza. & 0,2151 & 0,0038 \\
\hline & P855 Actividades de apoyo a la enseñanza. & 0,4514 & 0,0004 \\
\hline & Q861 Actividades de hospitales y clínicas. & 0,2600 & 0,0022 \\
\hline & Q862 Actividades de médicos y odontólogos. & 0,1387 & 0,0213 \\
\hline & Q869 Otras actividades de atención de la salud humana. & 0,1668 & 0,0048 \\
\hline & Q871 Actividades de atención de enfermería en instituciones. & 0,5995 & 0,0001 \\
\hline & Q872 Actividades de atención en instituciones para personas con $\mathrm{r}$ & 0,4447 & 0,0004 \\
\hline & Q873 Actividades de atención en instituciones para personas de ed & 0,4964 & 0,0003 \\
\hline & Q879 Otras actividades de atención en instituciones. & 0,3969 & 0,0005 \\
\hline & Q881 Actividades de asistencia social sin alojamiento para person & 0,4690 & 0,0002 \\
\hline & Q889 Otras actividades de asistencia social sin alojamiento. & 0,2515 & 0,0023 \\
\hline & R900 Actividades creativas, artísticas y de entretenimiento. & 0,3667 & 0,0008 \\
\hline & R910 Actividades de bibliotecas, archivos, museos y otras activid & 0,4240 & 0,0005 \\
\hline & R920 Actividades de juegos de azar y apuestas. & 0,2046 & 0,0048 \\
\hline & R931 Actividades deportivas. & 0,2127 & 0,0025 \\
\hline & R932 Otras actividades de esparcimiento y recreativas. & 0.2498 & 0,0026 \\
\hline & S941 Actividades de asociaciones empresariales, profesionales y d & 0,3036 & 0,0015 \\
\hline & S942 Actividades de sindicatos. & 0,4269 & 0,0006 \\
\hline & S949 Actividades de otras asociaciones. & 0,1664 & 0,0076 \\
\hline & S951 Reparación de computadoras y equipo de comunicaciones. & 0,1563 & 0,0048 \\
\hline & S952 Reparación de efectos personales y enseres domésticos. & 0,0974 & 0,0256 \\
\hline & S960 Otras actividades de servicios personales. & 0,0643 & 0,0364 \\
\hline
\end{tabular}

Tabla 12: Índice de especialización con relación a la media nacional (0.1452) 


\section{Agrupación de sectores económicos}

Se agrupa de acuerdo al algoritmo descrito en 20 grupos que se escribe a continuación.

\begin{tabular}{|c|c|}
\hline Grupos & Actividad económica \\
\hline 1 & $\begin{array}{l}\text { C101 Elaboración y conservación de carne. } \\
\text { C106 Elaboración de productos de molinería, almidones y productos } \\
\text { C107 Elaboración de otros productos alimenticios. } \\
\text { C141 Fabricación de prendas de vestir, excepto prendas de piel. } \\
\text { C162 Fabricación de hojas de madera para enchapado y tableros a b } \\
\text { C251 Fabricación de productos metálicos para uso estructural, tan } \\
\text { C310 Fabricación de muebles. } \\
\text { D351 Generación, transmisión y distribución de energía eléctrica. } \\
\text { E360 Captación, tratamiento y distribución de agua. } \\
\text { G452 Mantenimiento y reparación de vehículos automotores. } \\
\text { G454 Venta, mantenimiento y reparación de motocicletas y de sus p } \\
\text { G462 Venta al por mayor de materias primas agropecuarias y animal } \\
\text { G471 Venta al por menor en comercios no especializados. } \\
\text { G472 Venta al por menor de alimentos, bebidas y tabaco en comerci } \\
\text { G473 Venta al por menor de combustibles para vehículos automotore } \\
\text { G475 Venta al por menor de otros enseres domésticos en comercios } \\
\text { G476 Venta al por menor de productos culturales y recreativos en } \\
\text { G477 Venta al por menor de otros productos en comercios especiali } \\
\text { G478 Venta al por menor de puestos de venta y mercados. } \\
\text { H492 Otras actividades de transporte por vía terrestre. } \\
\text { I551 Actividades de alojamiento para estancias cortas. } \\
\text { I561 Actividades de restaurantes y de servicio móvil de comidas. } \\
\text { I563 Actividades de servicio de bebidas. } \\
\text { J601 Transmisiones de radio. } \\
\text { J602 Programación y transmisiones de televisión. } \\
\text { J619 Otras actividades de telecomunicaciones. } \\
\text { K641 Intermediación monetaria. } \\
\text { M691 Actividades jurídicas. } \\
\text { M742 Actividades de fotografía. } \\
\text { N821 Actividades administrativas y de apoyo de oficina. } \\
\text { O841 Administración del estado y aplicación de la política económ } \\
\text { O842 Prestación de servicios a la comunidad en general. } \\
\text { P851 Enseñanza preprimaria y primaria. } \\
\text { P852 Enseñanza secundaria. } \\
\text { P854 Otros tipos de enseñanza. } \\
\text { Q861 Actividades de hospitales y clínicas. } \\
\text { Q862 Actividades de médicos y odontólogos. } \\
\text { Q869 Otras actividades de atención de la salud humana. } \\
\text { Q889 Otras actividades de asistencia social sin alojamiento. } \\
\text { R910 Actividades de bibliotecas, archivos, museos y otras activid }\end{array}$ \\
\hline
\end{tabular}




\begin{tabular}{ll}
\hline Grupos & Actividad económica \\
\hline
\end{tabular}

R920 Actividades de juegos de azar y apuestas.

R931 Actividades deportivas.

R932 Otras actividades de esparcimiento y recreativas.

S941 Actividades de asociaciones empresariales, profesionales y d

S942 Actividades de sindicatos.

S949 Actividades de otras asociaciones.

S952 Reparación de efectos personales y enseres domésticos.

S960 Otras actividades de servicios personales.

2 C102 Elaboración y conservación de pescados, crustáceos y molusco

C103 Elaboración y conservación de frutas, legumbres y hortalizas

C105 Elaboración de productos lácteos.

C110 Elaboración de bebidas.

C131 Hilatura, tejedura y acabados de productos textiles.

C139 Fabricación de otros productos textiles.

C151 Curtido y adobo de cueros; fabricación de maletas, bolsos de

C152 Fabricación de calzado.

C161 Aserrado y acepilladura de madera.

C181 Impresión y actividades de servicios relacionados con la imp

C221 Fabricación de productos de caucho.

C222 Fabricación de productos de plástico.

C231 Fabricación de vidrio y productos de vidrio.

C239 Fabricación de productos minerales no metálicos n.c.p.

C259 Fabricación de otros productos elaborados de metal, activida

C275 Fabricación de aparatos de uso doméstico.

C282 Fabricación de maquinaria de uso especial.

C292 Fabricación de carrocerías para vehículos automotores; fabri

C293 Fabricación de partes, piezas y accesorios para vehículos au

C321 Fabricación de joyas, bisutería y artículos conexos.

C322 Fabricación de instrumentos musicales.

C323 Fabricación de artículos de deporte.

C324 Fabricación de juegos y juguetes.

C325 Fabricación de instrumentos y materiales médicos y odontológ

C331 Reparación de productos elaborados de metal, maquinaria y eq

F410 Construcción de edificios.

F432 Instalaciones eléctricas y de fontanería y otras instalacion

F433 Terminación y acabado de edificios.

F439 Otras actividades especializadas de construcción.

G451 Venta de vehículos automotores.

G453 Venta de partes, piezas y accesorios para vehículos automoto

G461 Venta al por mayor a cambio de una comision o por contrato.

G463 Venta al por mayor de alimentos, bebidas y tabaco.

G464 Venta al por mayor de enseres domésticos.

G465 Venta al por mayor de maquinarias equipos y materiales. 


\begin{tabular}{|c|c|}
\hline Grupos & Actividad económica \\
\hline & $\begin{array}{l}\text { G466 Otras actividades de venta al por mayor especializada. } \\
\text { G469 Venta al por mayor de otros productos no especializados. } \\
\text { G474 Venta al por menor de equipo de información y de comunicacio } \\
\text { G479 Venta al por menor no realizada en comercios, puestos de ven } \\
\text { H522 Actividades de apoyo al transporte. } \\
\text { H531 Actividades postales y de mensajería. } \\
\text { H532 Actividades de mensajería. } \\
\text { I562 Suministro de comidas por encargo y otras actividades de ser } \\
\text { J581 Publicación de libros, periódicos y otras actividades de pub } \\
\text { J592 Actividades de grabación de sonido y edición de música. } \\
\text { J611 Actividades de telecomunicaciones alámbrica. } \\
\text { K649 Otras actividades de servicios financieros, excepto las de s } \\
\text { K651 Seguros. } \\
\text { L681 Actividades inmobiliarias realizadas con bienes propios o ar } \\
\text { L682 Actividades inmobiliarias realizadas a cambio de una retribu } \\
\text { M692 Actividades de contabilidad, teneduría de libros y auditoria } \\
\text { M702 Actividades de consultoría de gestión. } \\
\text { M711 Actividades de arquitectura e ingeniería y actividades conex } \\
\text { M731 Publicidad. } \\
\text { M741 Actividades especializadas de diseño. } \\
\text { M749 Otras actividades profesionales, científicas y técnicas n.c. } \\
\text { M750 Actividades veterinarias. } \\
\text { N771 Alquiler de vehículos automotores. } \\
\text { N772 Alquiler de efectos personales y artículos de uso doméstico. } \\
\text { N773 Alquiler de otros tipos de maquinaria, equipo y bienes tangi } \\
\text { N791 Actividades de agencias de viajes y operadores turísticos. } \\
\text { N801 Actividades de seguridad privada. } \\
\text { N802 Actividades de servicios de sistemas de seguridad. } \\
\text { N823 Organización de convenciones y exposiciones comerciales. } \\
\text { N829 Actividades de servicios de apoyo a las empresas n.c.p. } \\
\text { P853 Enseñanza superior. } \\
\text { Q872 Actividades de atención en instituciones para personas con r } \\
\text { Q879 Otras actividades de atención en instituciones. } \\
\text { Q881 Actividades de asistencia social sin alojamiento para person } \\
\text { R900 Actividades creativas, artísticas y de entretenimiento. } \\
\text { S951 Reparación de computadoras y equipo de comunicaciones. }\end{array}$ \\
\hline $\begin{array}{l}4 \\
5\end{array}$ & $\begin{array}{l}\text { C104 Elaboración de aceites y grasas de origen vegetal y animal. } \\
\text { C192 Fabricación de productos de la refinación del petróleo. } \\
\text { C108 Elaboración de alimentos preparados para animales. }\end{array}$ \\
\hline & $\begin{array}{l}\text { C120 Elaboración de productos de tabaco. } \\
\text { C262 Fabricación de ordenadores y equipo periférico. }\end{array}$ \\
\hline
\end{tabular}




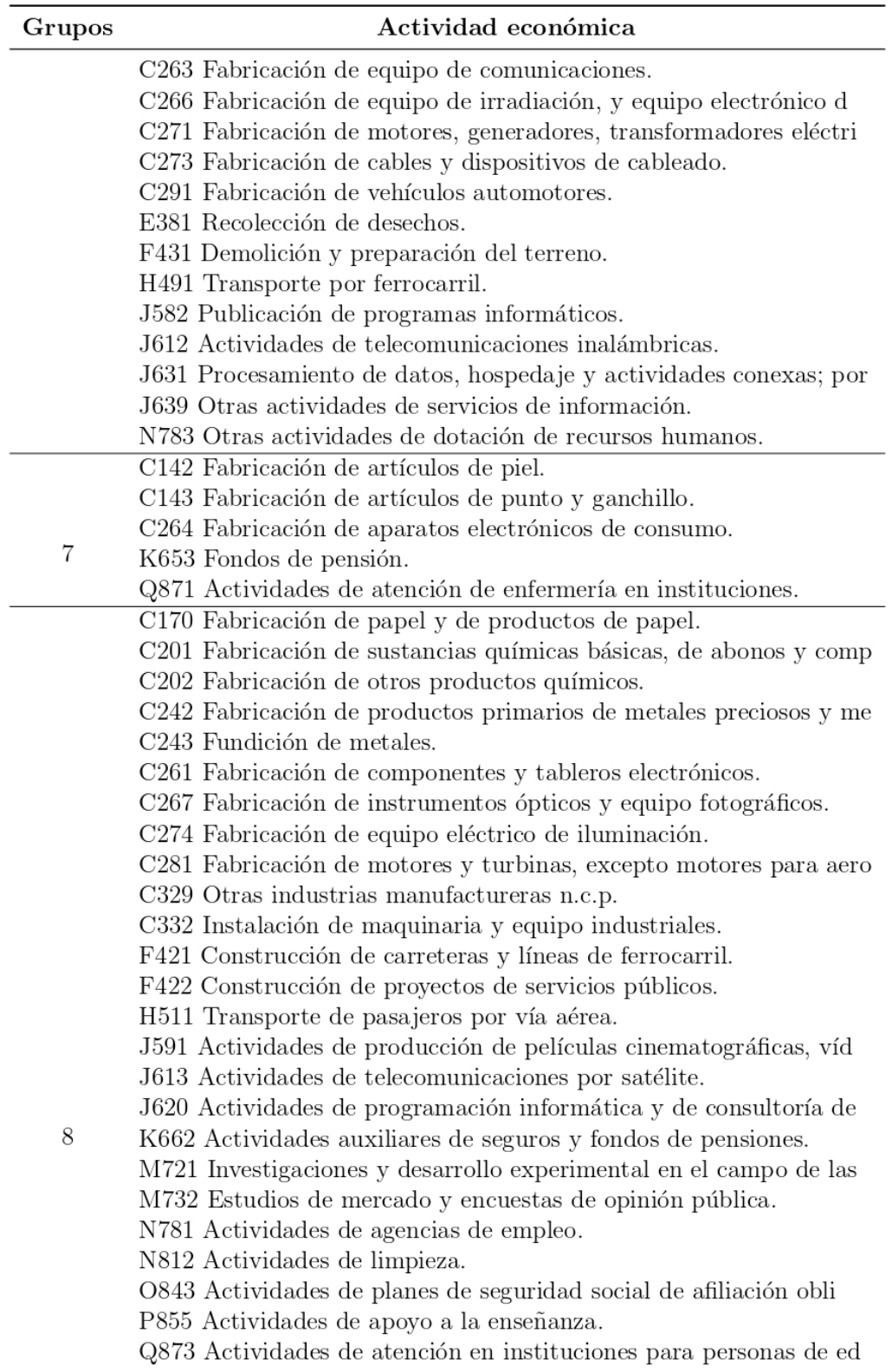




\begin{tabular}{|c|c|}
\hline Grupos & Actividad económica \\
\hline 9 & $\begin{array}{l}\text { C182 Reproducción de grabaciones. } \\
\text { C265 Fabricación de equipos de medición, prueba, navegación, cont } \\
\text { C279 Fabricación de otros tipos de equipo eléctrico. } \\
\text { K642 Actividades de sociedades de cartera. }\end{array}$ \\
\hline 10 & $\begin{array}{l}\text { C203 Fabricación de fibras artificiales. } \\
\text { C210 Fabricación de productos farmacéuticos, sustancias químicas } \\
\text { H501 Transporte marítimo y de cabotaje. } \\
\text { H502 Transporte por vías de navegación interiores. } \\
\text { H512 Transporte de carga por vía aérea. } \\
\text { H521 Almacenamiento y depósito. } \\
\text { K652 Reaseguros. } \\
\text { M712 Ensayos y análisis técnicos. }\end{array}$ \\
\hline 11 & $\begin{array}{l}\text { C241 Industrias básicas de hierro y acero. } \\
\text { K661 Actividades auxiliares de las actividades de servicios finan } \\
\text { K663 Actividades de gestión de fondos. } \\
\text { M701 Actividades de oficinas principales. } \\
\text { M722 Investigaciones y desarrollo experimental en el campo de las } \\
\text { N774 Arrendamiento de propiedad intelectual y productos similares } \\
\text { N782 Actividades de agencias de empleo temporal. } \\
\text { N799 Otros servicios de reservas y actividades conexas. } \\
\text { N813 Actividades de paisajismo y servicios de mantenimiento conex } \\
\text { N822 Actividades de centros de llamadas. } \\
\text { C252 Fabricación de armas y municiones. }\end{array}$ \\
\hline 12 & C252 Fabricación de armas y municiones. \\
\hline 13 & $\begin{array}{l}\text { C301 Construcción de buques y otras embarcaciones. } \\
\text { D353 Suministro de vapor y de aire acondicionado. }\end{array}$ \\
\hline & $\begin{array}{l}\text { C302 Fabricación de locomotoras y material rodante. } \\
\text { C309 Fabricación de equipo de transporte n.c.p. }\end{array}$ \\
\hline 14 & $\begin{array}{l}\text { F429 Construcción de otras obras de ingeniería civil. } \\
\text { K643 Fondos y sociedades de inversión y entidades financieras sim }\end{array}$ \\
\hline 15 & C303 Fabricación de aeronaves y naves espaciales y maquinaria con \\
\hline 16 & D352 Fabricación de gas; distribución de combustibles gaseosos po \\
\hline 17 & $\begin{array}{l}\text { E370 Evacuación de aguas residuales. } \\
\text { E383 Recuperación de materiales. } \\
\text { H493 Transporte por tuberías. } \\
\text { I552 Actividades de campamentos, parques de vehículos de recreo y }\end{array}$ \\
\hline $\begin{array}{l}18 \\
19 \\
20\end{array}$ & $\begin{array}{l}\text { E382 Tratamiento y eliminación de desechos. } \\
\text { E390 Actividades de descontaminación y otros servicios de gestió } \\
\text { I559 Otras actividades de alojamiento. } \\
\text { N803 Actividades de investigación. }\end{array}$ \\
\hline
\end{tabular}

Tabla 13: Agrupación media de sectores. Elaboración propia 


\section{Notas DE PÁGINA}

${ }^{1}$ Clasificación de empresas de acuerdo a la Resolución 1260 de la Comunidad Andina (CAN).

${ }^{2}$ (agricultura, minas, organizaciones y órganos extraterrtoriales)

${ }^{3}$ número de personas

${ }^{4}$ Clasificación Nacional de Actividades Económicas (CIIU REV. 4.0) a 3 dígitos

FECHA DE RECEPCIÓN DEL ARTÍCULO: 15 OCTUBRE DE 2018 FECHA DE ACEPTACIÓN: 30 SEPTIEMBRE DE 2019

VERSIÓN FINAL: 10 OCTUBRE DE 2019 


\section{Notas DE INVESTIGACIÓN}

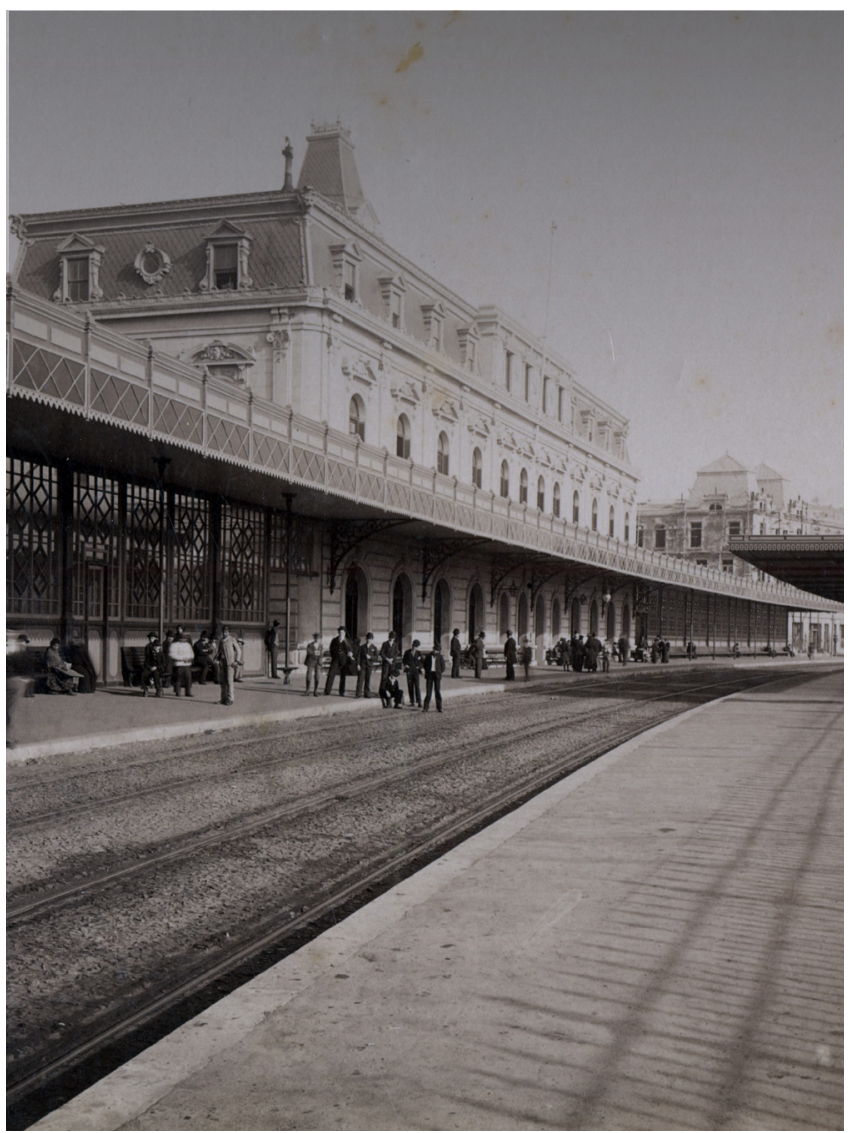


